\title{
Experience Sampling of the Degree of Mind Wandering Distinguishes Hidden Attentional States
}

\author{
Anthony P. Zanesco, Ekaterina Denkova, Joanna E. Witkin, \& Amishi P. Jha \\ ${ }^{1}$ Department of Psychology, University of Miami, Florida, U.S.A. \\ E-mail: apz13@miami.edu
}

\begin{abstract}
Experience sampling of attentional states has consistently demonstrated that mind wandering is a frequent and disruptive obstacle when one must sustain attention during continuous task performance. Yet, methods commonly used to assess the subjective experience of mind wandering may conflate several potential sources of meaningful variation in individuals' degree of task engagement. In the present study, we examined evidence for distinct and identifiable patterns in subjective reports of the degree of task-related attentional focus during a sustained attention task in a large sample of adults $(N=537)$. Experience sampling probes embedded in the task assessed task-related focus using a continuum of response ratings ranging from 1 (on-task) to 6 (off-task). Participants used a range of probe response options in categorizing their current attentional state, and the continuum of probe ratings differentiated patterns of behavioral performance in the moments preceding probes. Markov-chain modeling of the categorical time series sequence of probe ratings further revealed distinct and behaviorally relevant hidden states underlying probe rating behavior. We replicated these findings in two additional independent data sets. Collectively, these findings suggest that three or more hidden attentional states best account for subjective ratings of taskrelated focus. The implications of these findings for models of sustained attention and mind wandering are discussed.
\end{abstract}

Keywords: attention, experience sampling, hidden Markov model, mind wandering, task-unrelated thought

Experience sampling of subjective attentional states has remained an indispensable methodology in the study of mind wandering (Smallwood \& Schooler, 2006, 2015). Most commonly, studies have assessed mind wandering by embedding experience sampling probes within cognitive or perceptual tasks (Weinstein, 2018). When probed during task performance, individuals frequently report that their attention was directed towards something other than the task at hand (e.g., about half of probes; Kane et al., 2016). The prevalence of these off-task reports, and their negative correlation with behavioral performance (see for review, Randall, Oswald, \& Beier, 2014), is taken as evidence that mind wandering is pervasive and disruptive when one must sustain attention to meet performance goals. Yet, individuals likely draw on a range of distinct experiences to judge whether their attention is on or off-task making it possible that several attentional processes underlie on or off-task reports. Indeed, attentional lapses are multifaceted, resulting from variation in attentional control, arousal, effort, and task-unrelated thought (Esterman \& Rothlein, 2019). As such, examining systematic patterns of variation in probe-caught responses and their moment-to- moment links with behavior may be a valuable route by which to investigate the underlying attentional processes contributing to subjective reports.

Comprehensively characterizing the heterogeneity of experience sampling reports is important for understanding the attentional states contributing to sustained attentional performance. One aim of experience sampling in this domain is to account for the first-person experiences underlying lapses of attention, such as episodes of task-unrelated thought that contribute to performance errors but are not readily accessible through third-person measurement. It can be challenging, however, to introspect about one's own attentional state and categorically delineate one's experience of it in response to probes (Meier, 2018). Accordingly, individuals engage in a tension between choosing the most accurate response option compared to a merely satisfactory one (Krosnick, 1999; Weinstein, 2018). Further, they likely differ in the criterion with which they judge their current attention to be on or off-task based on the specific experience sampling methodology that is employed. 
Researchers frequently require individuals to make dichotomous judgments indicating either complete engagement or complete disengagement in their task (i.e., on-task or off-task) as a measure of mind wandering (Weinstein, 2018). Two recent studies provided evidence that requiring individuals to make such forced-choice judgments about the current state of their attention limits assessment of meaningful variation in different subjective experiences of attentional states and inflates estimates of off-task reports in the dichotomous probe procedure (Robison, Miller, \& Unsworth, 2019; Seli et al., 2018a). Seli et al. (2018a) compared participants' answers on dichotomous probes to those using a continuous Likert-like 5-point scale when randomly probed about the focus of their attention throughout their day. While participants reported being offtask $40 \%$ of the time in response to dichotomous probes, only $12 \%$ of probes were rated as being highly off-task in the continuous rating condition. This disparity suggests that estimates of off-task reports are inflated in dichotomous probe procedures. In line with these findings, Robison et al. (2019) demonstrated that individuals conflate instances of task-related interference, external distraction, and mindblanking, with episodes of task-unrelated thought, when given only two categorical response options. Together, these studies suggest that individuals experience a range of distinct mental phenomena that contribute to their subjective reports of attentional lapses. However, when given limited choices for categorizing their attentional focus, individuals may classify these diverse phenomena as belonging to the same category of experiences.

Such findings have implications for understanding the nature of sustained attention and mind wandering in daily life, as well as during cognitively demanding tasks. They also support the notion that individuals experience and are able to report on subtler fluctuations in their attention than can be obtained from forced-choice dichotomous reporting. Studies have also found subtler variations in on and off-task reports to be behaviorally meaningful. Indeed, linear associations have been found between task performance in the moments preceding probes and the degree to which individuals report being off-task using continuous ratings (i.e., Laflamme, Seli, \& Smilek, 2018; Hawkins, Mittner, Forstmann, \& Heathcote, 2019). That is, gradual increases in the degree to which individuals report being off task corresponds to increasing reaction time variability. Nonetheless, little is known about the attentional states, or the collection of distinct attentional processes, that contribute to variability in task performance and underlie experience sampling ratings of individuals' degree of task-related focus.

Investigating the nature of these attentional states, including how many of such states can be meaningfully identified, is needed to understand the attentional processes measured with experience sampling (cf. construct continuum specification; Tay \& Jebb, 2018). Importantly, the correspondence between task performance on trials directly preceding experience sampling probes and probe ratings at that moment may differentiate attentional states. Linear associations between task performance and probe ratings would suggest that individuals distinguish among diverse but behaviorally relevant attentional fluctuations (i.e., multiple 'states'; see e.g., Laflamme et al., 2018). Yet, past studies have not examined whether non-linear associations may be present in addition to linear effects. It is instead possible that non-linear associations exist between off-task ratings and performance, implicating only highly off-task ratings in moments of task disengagement and mind wandering. Furthermore, the frequency distribution of probe rating responses can provide evidence for whether individuals utilize the full continuum of probe ratings or naturally divide their experience into two distinct kinds of attentional experiences. If probe ratings primarily reflect continuous gradations in individuals' taskrelated focus, the overall distribution of probe rating responses ought to range across the full continuum of response options (cf. Seli et al., 2018a). But prior studies have not fully characterized probe response distributions in the context of sustained attention tasks. Nonetheless, while limited, the evidence examining probes' correspondence with behavior (e.g., Laflamme et al., 2018; Hawkins et al., 2019), as well as the frequency distribution of probes (e.g., Seli et al., 2018a; Robison et al., 2019), both suggest that the attentional states underlying probe responses may not be dichotomous.

In addition, examining the temporal patterns of systematic transitions between probe ratings may provide insight into underlying attentional states during sustained attention tasks. While the meandering and spontaneous nature of thought has been argued to be central to definitions of mind wandering (Christoff, Irving, Fox, Spreng, \& Andrews-Hanna, 2016; Irving, 2016; Irving \& Thompson, 2018), few studies have directly characterized dynamic patterns in self-reported ratings of attentional focus or mind wandering with methods that can account for the temporal ordering of probe reports over time. Some researchers have begun to investigate these questions in more detail by exploring whether mind wandering occurs randomly during task performance or whether its frequency and pattern of occurrence may be periodic, depending perhaps, on how attention is regulated over time (e.g., Zanesco, 2020). As one example, studies have provided evidence for systematic change in the frequency of mind wandering with greater time-on-task (e.g., Krimsky, Forster, Llabre, \& Jha, 2017; Thomson, Seli, Besner, \& Smilek, 2014).

Various attentional phenomena are therefore understood according to their temporal dynamics (e.g., time-on-task 
effects; Esterman \& Rothlein, 2019). The underlying structure of individuals' attentional experience may be elucidated based on systematic patterns in the temporal sequences of probe ratings. One novel approach is to employ Markov-chain modeling. Hidden Markov models of probes assume that future probe ratings probabilistically depend on current ratings with unobservable (i.e., hidden) states that govern the distribution of observed probe ratings at each moment in time (Visser, Raijmakers, \& Molenaar, 2002). Hidden Markov models therefore characterize transition probabilities between probes from one moment to the next and can identify the most probable hidden states responsible for the complex and varied probe rating behavior reported during cognitive task performance. Hidden Markov models have been used previously to distinguish on from off-task states based on variability in reaction times (Bastian \& Sackur, 2013). While important, this past work did not apply these models directly to experience sampling probes and did not attempt to identify the number of hidden states present during task performance. If individuals primarily divide their experiences into distinct states of complete task engagement and mind wandering, two hidden states ought to adequately describe the probe rating sequences. More than two hidden states, however, would argue that continuous ratings of individuals' task-related focus cannot be reduced to a dichotomous process. Markov models may therefore provide key insights into the attentional states underlying probe responses and complement examination of probe correspondence with performance and probe report frequency.

In the present study, we sought to characterize the attentional states underlying experience sampling probe ratings. Our central question was to determine whether probe ratings during a sustained attention task best reflect dichotomous episodes of task engagement versus disengaged mind wandering, or whether they reflect meaningful gradations in task-related focus. Five hundred and thirty-seven adults completed the Sustained Attention to Response Task (SART; Robertson, Manly, Andrade, Baddeley, \& Yiend, 1997) with 28 probe questions interspersed throughout task performance assessing the degree of their task-related focus along a continuous Likert-like scale. Three approaches were employed. We examined the linear and non-linear correspondence between experience sampling reports and behavioral task performance, the frequency and temporal sequence of probe ratings, and their underlying hidden Markov states. Furthermore, we examined whether patterns of transition probabilities and hidden Markov states generalized to other task contexts by conducting secondary analyses of deidentified participant data from two independent studies conducted by Goller, Banks, and Meier (2020), and Wiradhany, van Vugt, and Nieuwenstein (2019). These studies were chosen because they contained large samples of participants, utilized continuous Likert-like ratings of the degree of task focus, and provided welldocumented deidentified data online

\section{Methods}

We report how we determined our sample size, all data exclusions, all manipulations, and all measures in the study.

\section{Participants}

Five hundred and thirty-seven United States military service members were included as participants. Participants were predominantly male ( $n=24$ female) adults aged 28.59 years on average $(S D=8.079$, range $=18-54)$. Data were aggregated from participants who were recruited as part of a series of studies investigating the implementation of cognitive wellness and resilience interventions in the military, but all data in the present study were collected prior to random assignment to any intervention arm. The sample size of the present study was not determined in advance. Instead, the sample size was based on the total aggregated number of participants who took part in this series of studies and completed the SART. All participants provided informed consent in compliance with the Institutional Review Board of the University of Miami. All testing occurred during participants' military duty day. Participants were therefore not compensated beyond their wages for participation in the project per Department of Defense regulations regarding service member compensation during the duty day.

\section{Procedure}

A series of computerized cognitive tasks and questionnaires were administered to participants. One task in this series, which is of selective interest herein, was the Sustained Attention to Response Task (SART; Robertson et al., 1997). The SART was the first of two tasks for 357 participants, and the second of three tasks for the remaining 180 participants. The SART was the only cognitive task with embedded experience sampling probes. The other tasks were a Stroop task and a working memory delayed recognition task. Testing was administered by a team of 1 to 3 experimenters in a group setting of up to 16 participants. Each session lasted approximately one to two hours and took place in a quiet classroom on the participants' military installation. Each participant was seated approximately $57 \mathrm{~cm}$ from their own PC laptop, and stimuli were presented via E-prime (Version 2.0; Psychology Software Tools, Pittsburgh, PA).

\section{Sustained Attention to Response Task (SART).}

Participants were administered a modified version of the SART (see Figure 1 for task schematic). Single digits (0 through 9) were continuously presented on screen one at a 


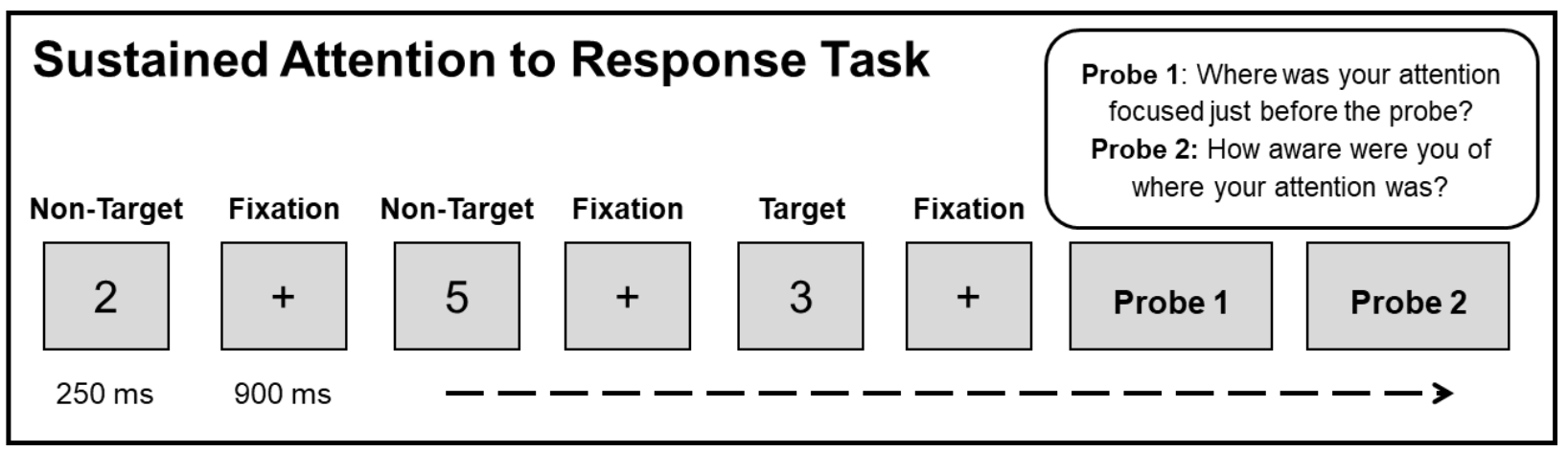

Figure 1: Schematic demonstrating the design of the Sustained Attention to Response Task (SART). Single digits (0 through 9) were continuously presented on screen one at a time for $250 \mathrm{msec}$ followed by an inter-trial-interval of $900 \mathrm{msec}$ during which a fixation cross was presented. Participants were instructed to refrain from pressing the spacebar to the target number 3 (5\% of trials) and to press the spacebar for all other non-target digits. Experience sampling probes periodically interrupted task performance to ask participants to respond to two probe questions using a Likert-like 6-point scale.

time for $250 \mathrm{msec}$. Each digit was followed by an inter-trialinterval of $900 \mathrm{msec}$ during which a fixation cross was presented. Participants were instructed to refrain from pressing the spacebar to the number 3 (target) and to press the spacebar for all other numerical digits (non-targets) while emphasizing both accuracy and speed. Stimuli were presented in black font on a gray screen, and responses were recorded during the stimulus display or the inter-trial interval. Participants first completed a 163-trial practice block (146 non-targets, 6 targets, and 11 sets of probes), during which experimenters were available to answer any remaining questions from participants regarding the task instructions. Participants then completed two experimental blocks, which consisted of a total of 519 non-targets, 27 targets, and 28 sets of probes. Trial order was quasirandomized so that targets were always separated by at least one other non-target digit.

On probe trials, participants responded to two consecutive probe questions in order to assess their current attentional state. Probes were randomly dispersed throughout the task, separated by 19.5 trials on average $(S D=9.72$, range $=4$ 37 ). The probe questions occurred on the same trials during the task for all participants. Participants were instructed that probe questions will occasionally ask about the focus of their attention. The first probe question (attentional probe) asked, "Where was your attention focused just before the probe?" with participants responding using a 6-point Likert-like scale ranging from 1 (on-task) to 6 (off-task). The second question (awareness probe) asked, "How aware were you of where your attention was?" with participants responding from 1 (aware) to 6 (unaware). The questions were displayed until a response was provided.

Results from the practice block were not included in any analyses. Responses to the 28 awareness probes (probe question 2) were also not considered or analyzed herein. Several dependent measures were calculated to summarize behavioral performance on the SART. Accuracy was indexed by $A^{\prime}$, a nonparametric measure of detection sensitivity (see Stanislaw \& Todorov, 1999, for calculations). Reaction time variability was assessed using the intra-individual coefficient of variation (ICV), which was calculated as the standard deviation of reaction time (RT) for correct non-target trials divided by the mean RT of correct non-target trials for each participant. Greater ICV reflects more variation in response time. Furthermore, behavioral performance preceding each probe was indexed by calculating non-target accuracy and ICV for sets of up to 5 non-target trials preceding each probe. ICV was only calculated for probes that were preceded by correct trials (i.e., responding to all non-target digits). Participants had an average of $24.12(S D=5.02$, range $=3-$ 28) sets of trials preceding probes available for calculation of ICV.

\section{Analyses}

The association between non-target accuracy, ICV, and the rating (from 1 to 6 ) of each of the 28 attentional probes were examined using mixed effects models with PROC Mixed in SAS 9.4. Linear and quadratic associations between probe ratings and preceding trial performance were examined. Parameters were estimated using maximum likelihood estimation, and models describe the effects in terms of an intercept (i.e., starting point) and linear or quadratic slope (i.e., rate of change), with random effects representing between-person variability in these parameters. The intercept was centered at probe ratings of 1 (on-task). Individuals' mean probe rating, representing the additional contextual effect of individuals (level 2) average probe rating on task performance, was also included in models to allow for separate examination of within-person and between-person associations. Random intercepts and linear slopes were included to allow for variability between individuals and slopes respectively. We excluded 16 participants (3\% of all participants) from all analysis if their testing session was interrupted or incomplete, or if they were observed by the 

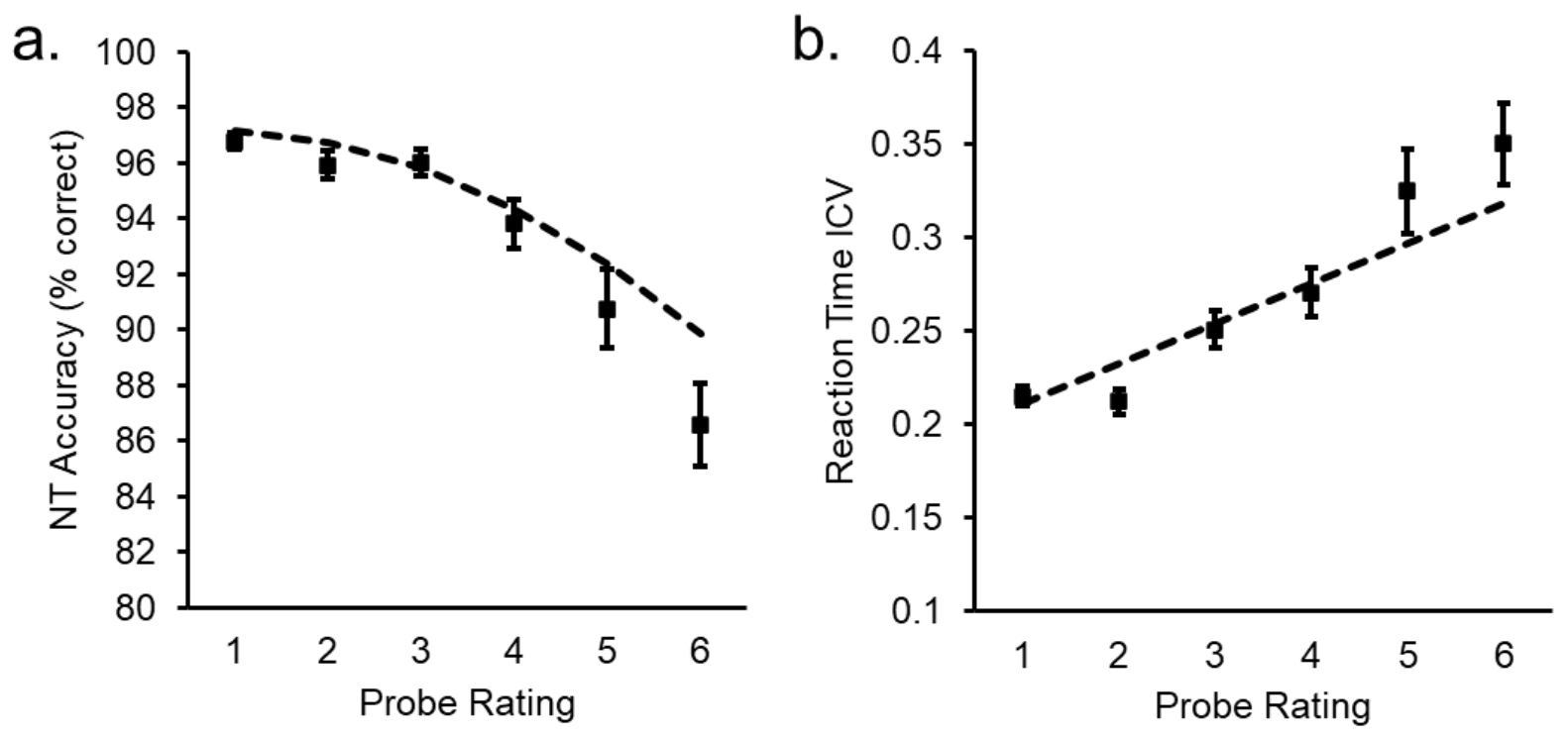

Figure 2: Mixed effects model estimated associations between probe ratings (rated from 1 "on-task" to 6 "off-task") and (a.) non-target (NT) accuracy (\% trials correct) and (b.) reaction time ICV for trials preceding experience sampling probes are depicted as slopes, alongside observed means and standard errors for each binned probe rating category.

experimenter to not comply with task instructions, selfreported that they did not follow instructions, or were observed to be falling asleep during assessment. In addition, 7 participants $(1.3 \%$ of all participants) were excluded because their $A^{\prime}$ was $3 S D$ below the grand mean $\left(A^{\prime}<.349\right)$ of the remaining participants. Following exclusions, there was a total of 514 participants with data included in all reported analyses.

Markov-chain analysis of participant's attentional probe ratings considered each rating as a discrete categorical state that occurred in sequence across the duration of the SART. Markov-chain transition probabilities were calculated based on the entire sample of probe rating sequences in $R$ using package seqHMM (Helske \& Helske, 2019). Each row of the transition probability matrix reflects the total probability (i.e., each row sums to 1) of each probe rating transitioning to each other rating as observed from the probe rating sequences. Transition probabilities were compared to a null distribution of probabilities occurring by chance using nonparametric permutation-based statistical comparisons. Probe ratings were shuffled randomly in time for each individual for 5000 iterations and an empirical null distribution of transition probabilities occurring by chance were calculated. The actual transition probabilities were compared to the empirical distribution of 5000 permutations to identify whether the observed probability was sufficiently extreme (greater than or less than $97.5 \%$ of all permutations) with respect to the null distribution of probabilities occurring by chance.
We further characterized the hidden response generation processes underlying temporal patterns of attentional probe ratings by estimating hidden Markov states based on the entire sample of probe rating sequences (Helske \& Helske, 2019). Each hidden state is characterized by an initial probability of occurrence that indicates the likelihood of a sequence beginning with that hidden state, as well as transition probabilities that indicate the probability of a hidden state transitioning to other hidden states. Hidden states are further characterized by emission probabilities that indicate the probability of that hidden state "emitting" the observed probe rating responses. Emission probabilities govern the distribution of observed probe ratings at each moment in time based on the hidden state at that moment. We examined the trial-level association between hidden states and non-target accuracy and ICV, calculated from trials preceding probe ratings, using linear mixed effects models.

The optimal number of hidden states were determined based on comparison of the Bayesian Information Criterion (BIC) between hidden Markov models calculated for different numbers of hidden states. The hidden Markov model with the fewest hidden states that had the lowest BIC was retained as the optimal model. This was determined by choosing the number of hidden states such that adding additional hidden states did not greatly reduce the BIC compared to earlier state solutions (i.e., "the elbow criterion"). Empirical validation of the optimal number of hidden states was then conducted by simulating 5000 sets of 500 sequences of 28 probe ratings based on the initial probability of occurrence, hidden state transition probabilities, and emission 
Table 1: Distribution of probe rating responses and descriptive statistics for probe-related behavioral performance

\begin{tabular}{lcccccc}
\hline & \multicolumn{6}{c}{ Probe Rating } \\
\cline { 2 - 7 } & 1 & 2 & 3 & 4 & 5 & 6 \\
\hline \% Total Probes & 63.10 & 15.28 & 8.61 & 4.26 & 2.67 & 6.09 \\
Non-Target Accuracy & $96.77(6.54)$ & $95.92(9.41)$ & $96.00(8.07)$ & $93.81(12.01)$ & $90.77(16.01)$ & $86.59(17.96)$ \\
ICV & $0.215(0.11)$ & $0.212(0.12)$ & $0.251(0.16)$ & $0.271(0.18)$ & $0.325(0.23)$ & $0.350(0.24)$ \\
\hline
\end{tabular}

Note: The percentage of all probe ratings classified as a particular probe rating response (1 “on-task" to 6 "off-task") are provided. Means and standard deviations for non-target accuracy (\% correct) and ICV are calculated for individuals from trials preceding each probe rating response.

probabilities of different hidden state solutions, to examine how well each of the hidden state solutions recreated the transition probabilities of the empirical data. Correlations between the transition probability matrix of the observed probe rating data and each of the 5000 simulated sets of sequences were calculated for different numbers of hidden states, and the distribution of correlations were compared between hidden state solutions.

\section{Results}

We first examined descriptive statistics of behavioral measures derived from the SART. $A^{\prime}$ was 0.814 (SD = 0.140 ), and participants correctly detected and withheld their response to $47.76 \%(S D=25.81)$ of target stimuli on average. Mean reaction time in response to non-target stimuli was $324.94 \mathrm{msec}(S D=88.51)$ on average, and reaction time ICV was $0.404(S D=0.226)$ on average. Mean rating for the attentional probe (ratings from 1 "on task" to 6 "off task") was $1.864(S D=1.034)$, whereas the median was 1.446 and the mode was 1 .

Correlations between individuals' mean probe rating and behavioral performance demonstrated a significant negative correlation between mean probe rating and $A^{\prime}, r=-0.287, p<$ $.001,95 \%$ CI [-0.364, -0.206], and a significant positive correlation between mean probe rating and ICV, $r=0.248, p$ $<.001,95 \%$ CI $[0.165,0.327]$. There was no significant correlation between mean attentional probe rating and nontarget reaction time, $r=-0.044, p=.316,95 \%$ CI [-0.130, $0.042]$. Individuals who self-reported being more off-task on average had lower accuracy $\left(A^{\prime}\right)$ and had greater variability (ICV) in their response times.

\section{Probe-related Behavioral Performance}

We examined the association between probe ratings and behavioral performance (non-target accuracy and ICV) for trials preceding each respective probe response. Descriptive statistics from individuals for probe-related behavioral performance are reported in Table 1. Parameters from linear mixed effects models are reported in Table 2. A mixed effects model revealed no significant linear change in accuracy as participants reported being increasingly off-task ( $b=-0.154, p=.578,95 \%$ CI $[-0.697,0.388])$, but there was a significant quadratic effect indicating that the rate of change negatively accelerated by $-0.262 \%(p<.001,95 \% \mathrm{CI}$ $[-0.416,-0.109])$ for each probe rating from 1 to 6 . The contextual level-2 effect was not significant $(b=0.567, p=$ $.064,95 \%$ CI $[-1.165,0.032])$, suggesting that trial-level fluctuations in probe ratings primarily contributed to the accuracy of preceding responses instead of differences at the person level. Thus, the negative association between nontarget accuracy and probe ratings was stronger on trials in which individuals reported being more off-task. There was little change in non-target accuracy in trials preceding "ontask" probe ratings (i.e., ratings 1 to 4), but a large decrease in accuracy when individuals reported being highly off-task (see Figure 2a).

There was also a significant linear effect of probe rating on individuals' ICV for trials preceding their probe response, such that probes had greater ICV on preceding trials by 0.021 $(p<.001,95 \%$ CI $[0.017,0.026])$ for each probe rating from 1 to 6 (see Table 2 for model parameters). The contextual level-2 effect was not significant $(b=0.003, p=.547,95 \%$ CI $[-0.008,0.014])$, suggesting that trial-level fluctuations primarily contributed to the variability of preceding responses instead of person-level differences. There was no significant quadratic effect $(b<0.001, p=.794,95 \%$ CI [$0.002,0.003])$, when the quadratic parameter was added to the model. ICV of trials preceding experience sampling probes was thus expected to increase linearly as individuals rated their attentional state as more off-task (see Figure 2b).

Consistent with prior literature (e.g., Krimsky et al., 2017), we also confirmed time-ordered linear changes in performance and probe ratings over the course of task performance with mixed effects models. Probe trial order was included as a fixed effect centered to the first probe trial, alongside random intercepts and linear slopes for participants. Non-target accuracy declined by $-0.093 \%$ ( $p<$ $.001,95 \% \mathrm{CI}[-0.126,-0.061])$ in the trials preceding probes for each of the 28 probes that were presented over the task. Similarly, ICV increased by $0.002(p<.001,95 \%$ CI $[0.0014,0.0025])$ each probe trial. Finally, mean probe rating 
Table 2: Model estimates of probe-related behavioral performance

\begin{tabular}{lcc}
\hline Model Parameter & Non-Target Accuracy & ICV \\
\hline Fixed Effects & $97.173(0.345)^{* * *}$ & $0.211(0.006)^{* * *}$ \\
$\quad$ Intercept & $-0.154(0.277)$ & $0.021(0.002)^{* * *}$ \\
Linear Probe Rating & $-0.262(0.078)^{* * *}$ & - \\
$\quad$ Quadratic Probe Rating & $-0.567(0.305)$ & $0.003(0.006)$ \\
Mean Probe Rating & & \\
Random Effects & $29.378(2.253)$ & $0.010(0.001)$ \\
$\quad$ Intercept variance & $0.515(1.718)$ & $0.001(0.000)$ \\
Linear Slope variance & $0.392(0.122)$ & - \\
Quadratic Slope variance & $7.070(1.757)$ & $0.001(0.000)$ \\
Intercept-Linear covariance & $-1.411(0.440)$ & - \\
Intercept-Quadratic covariance & $-0.388(0.426)$ & - \\
Linear-Quadratic covariance & $106.00(1.293)$ & $0.024(0.000)$ \\
Residual variance & 109488.5 & -9677.1 \\
BIC & 14392 & 12397 \\
\# Observations & 514 & 514 \\
Subjects $(N)$ & &
\end{tabular}

Note: Maximum likelihood estimates are reported for mixed effects models of non-target accuracy (\% correct) and reaction time ICV calculated from the trials preceding each probe rating response. Linear and quadratic effects of probe rating response are provided. These parameters represent the within-person (level 1) association between probe ratings and task performance. The mean probe rating parameter represents the additional contextual effect of individuals (level 2) average probe rating on task performance. Ratings are centered with respect to ratings of $1=$ "on-task." The number of subjects $(\mathrm{N})$ and observations contributing to the analyses are provided. Standard errors are reported in parentheses. ** $\mathrm{p}<.01$, ***p $<$ .001 .

increased by $0.021(p<.001,95 \%$ CI $[0.017,0.026])$ each probe trial. These findings indicate linear decreases in performance alongside increases in ICV and off-task probe ratings as probes were presented over time.

\section{Frequency Distribution of Attentional Probe Ratings}

We next examined evidence for a continuum of subjective attentional states reflected in the distribution of probe rating behavior. Table 1 reports the total distribution of attentional probe ratings for each rating category and descriptive statistics for non-target accuracy and ICV derived from trials preceding experience sampling probes. Figure 3 depicts the sequences of attentional probe ratings (from 1 "on-task" to 6 "off-task") during the SART for all individuals. The distribution of all 14393 probe ratings (see Figure 3b) was strongly positively skewed (skewness $=1.752$ ) and leptokurtic (kurtosis $=5.020$ ) with $63.10 \%$ of all probe ratings rated equal to 1 (on-task). Only $36.90 \%$ of all probes were rated greater than $1.13 .01 \%$ of probes were rated 4 or greater, and $6.09 \%$ of all probes were rated 6 (off-task). Participants on average used $3.07(S D=1.57,95 \%$ CI [2.936, 3.208], range $=1-6$ ) unique categories of probe rating responses. Thus, as a whole, participants used a range of responses when categorizing their task-related focus. Individuals overall were highly likely to report being on-task when randomly probed, and unlikely to report being off-task to some extent (i.e., a probe rating of 4,5 , or 6 ).

\section{Sequence Analysis of Attentional Probe Ratings}

Transition probabilities. Figure $3 \mathrm{c}$ depicts the transition probability matrix of all attentional probe ratings. Participants on average had $8.33(S D=6.80,95 \%$ CI [7.743, $8.918]$ ) transitions between distinct probe rating categories. The Markov-chain transition probabilities indicated that individuals were highly likely to report the probe rating they last reported, as indicated by strong transition probabilities along the diagonal of the transition probability matrix (see Figure 3c). Transitions were more likely to occur than chance (i.e., transition probabilities more extreme than $97.5 \%$ of 5,000 random permutations of probe rating sequences) for transitions between ratings of $1 \rightarrow 1,2 \rightarrow 2,3 \rightarrow 3,4 \rightarrow 4,5$ $\rightarrow 5$, and $6 \rightarrow 6$. Transitions were also more likely than chance to occur for transitions between ratings of $4 \rightarrow 5$ and $5 \rightarrow 4$. Individuals were also likely $(84.1 \%)$ to remain in a highly focused state following a report of being on-task (i.e., a probe rating of 1 ), as transitions from ratings of $1 \rightarrow 2$, 
a.

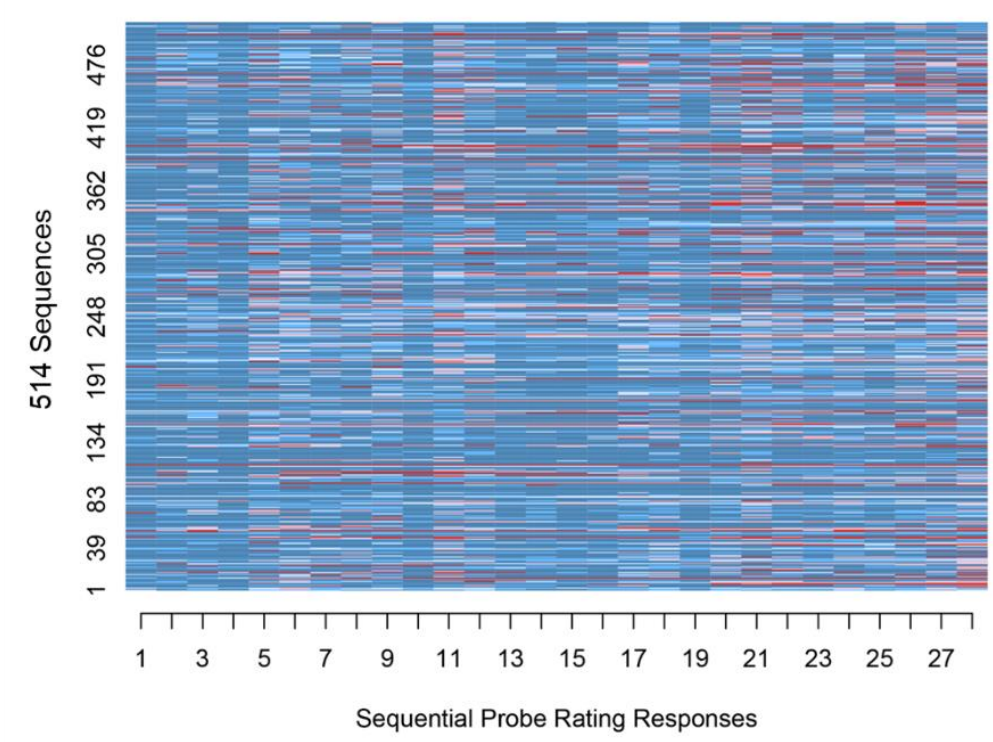

1. On-task $\square$ 3. Slightly On-task $\square$ 5. Moderately Off-task b.
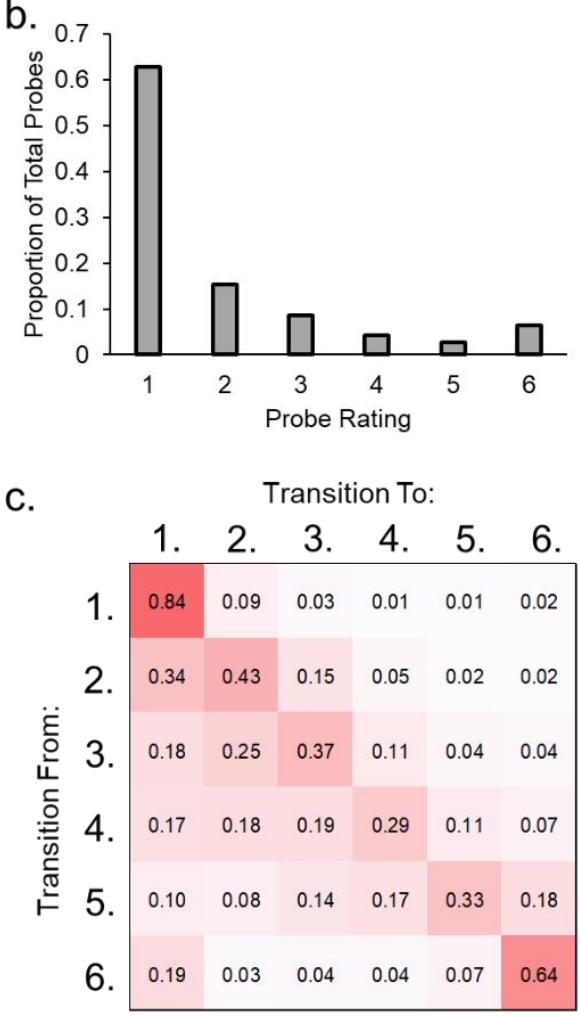

Figure 3: Sequences of 28 probe ratings (rated from 1 "on-task" to 6 "off-task") during the SART are depicted (a.) on rows for each individual ( $n=514$ ) sorted randomly. The distribution of all 14393 probe ratings (b.) and the transition probability matrix of probe ratings (c.) are shown for sequences of probe ratings. The transition probability matrix indicates the total probability (rows sum to 1) of all transitions from a probe rating to each other probe rating based on all sequences.

a.

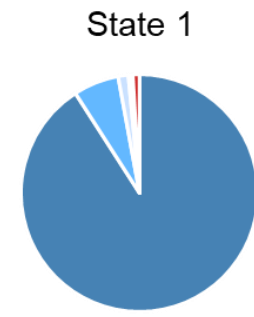

$81.86 \%$
State 2

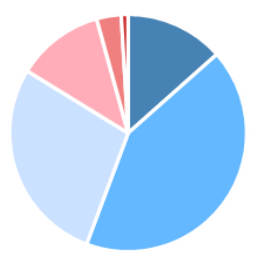

$15.61 \%$
State 3

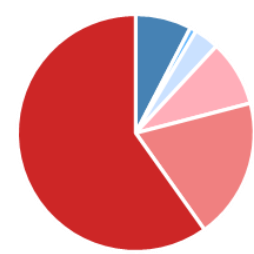

$2.52 \%$
1. On-task 2. Moderately On-task $\square$ 4. Slightly Off-task
5. Moderately Off-task 6. Off-task b. Transition To:

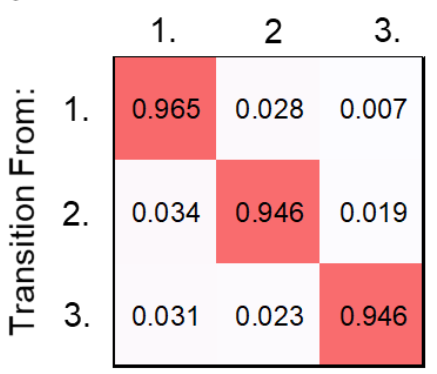

C. 35000

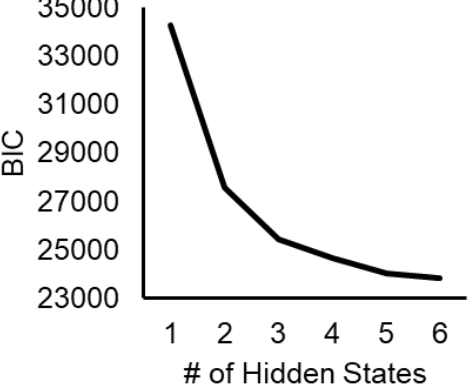

Figure 4: Three hidden Markov states were identified as the optimal model from the probe rating sequences. Each state is depicted (a.) as a circle chart in which the emission probabilities of each hidden state emitting probe ratings ( 1 "on-task" to 6 "offtask") are given as proportions of each state. Initial state probabilities are indicated below each circle chart. Transition probabilities between hidden states are indicated (b.) in the transition matrix. The Bayesian Information Criterion (BIC) is given (c.) for hidden Markov models with different numbers of hidden state solutions. 
Table 3: Hidden Markov models of probe rating sequences for different hidden state solutions

\begin{tabular}{|c|c|c|c|c|c|c|c|c|c|c|c|c|}
\hline \multirow[b]{2}{*}{ Model } & \multirow[b]{2}{*}{ BIC } & \multirow[b]{2}{*}{$\begin{array}{l}\text { Initial } \\
\text { Prob. }\end{array}$} & \multicolumn{4}{|c|}{ Transition Prob. } & \multicolumn{6}{|c|}{ Emission Prob. } \\
\hline & & & 1 & 2 & 3 & 4 & 1 & 2 & 3 & 4 & 5 & 6 \\
\hline 2 States & 27584.2 & & & & & & & & & & & \\
\hline State 1 & & 0.841 & 0.969 & 0.031 & & & 0.889 & 0.076 & 0.017 & 0.006 & 0.001 & 0.010 \\
\hline State 2 & & 0.159 & 0.032 & 0.968 & & & 0.091 & 0.313 & 0.231 & 0.119 & 0.080 & 0.167 \\
\hline 3 States & 25416.3 & & & & & & & & & & & \\
\hline State 1 & & 0.819 & 0.965 & 0.028 & 0.007 & & 0.909 & 0.062 & 0.014 & 0.005 & 0.001 & 0.009 \\
\hline State 2 & & 0.156 & 0.034 & 0.946 & 0.019 & & 0.135 & 0.423 & 0.280 & 0.119 & 0.034 & 0.009 \\
\hline State 3 & & 0.025 & 0.031 & 0.023 & 0.946 & & 0.076 & 0.009 & 0.034 & 0.090 & 0.192 & 0.598 \\
\hline 4 States & 24632.8 & & & & & & & & & & & \\
\hline State 1 & & 0.517 & 0.973 & 0.016 & 0.002 & 0.010 & 0.971 & 0.010 & 0.004 & 0.002 & 0.001 & 0.012 \\
\hline State 2 & & 0.361 & 0.010 & 0.946 & 0.043 & 0.001 & 0.655 & 0.251 & 0.061 & 0.023 & 0.006 & 0.004 \\
\hline State 3 & & 0.097 & 0.005 & 0.020 & 0.950 & 0.025 & 0.047 & 0.386 & 0.339 & 0.162 & 0.052 & 0.013 \\
\hline State 4 & & 0.025 & 0.029 & 0.009 & 0.021 & 0.941 & 0.082 & 0.008 & 0.027 & 0.057 & 0.182 & 0.645 \\
\hline
\end{tabular}

Note: Initial starting probabilities, transition probabilities among hidden Markov states, and emission probabilities of each hidden state emitting probe ratings ( 1 "on-task" to 6 "off-task"), are given for two hidden state, three state, and four state Markov models. The Bayesian Information Criterion (BIC) is indicated as a measure of model fit for each Markov model.

$1 \rightarrow 3,1 \rightarrow 4$, and $1 \rightarrow 5$, occurred less frequently than expected by chance alone.

After experiencing some degree of attentional drift away from the task, individuals were less likely than chance to transition from ratings of $2 \rightarrow 1,3 \rightarrow 1,4 \rightarrow 1,5 \rightarrow 1$, and 6 $\rightarrow 1$. Following a complete attentional lapse (i.e., probe rating of 6$)$, individuals were highly likely $(64.1 \%)$ to remain in that same off-task state during the next probe question, and were less likely than chance to transition to more on-task states for transitions between ratings of $6 \rightarrow 1,6 \rightarrow 2,6 \rightarrow$ 3 , and $6 \rightarrow 4$. Although infrequent, highly unfocused off-task states were therefore likely to linger or reoccur over time in contrast to other probe ratings. Transitions were also less likely than chance to occur for transitions between ratings of $2 \rightarrow 5,2 \rightarrow 6,4 \rightarrow 3$, and $5 \rightarrow 2$. All other transitions were no more or less likely to occur compared to chance alone. Transitions appeared highly asymmetric; individuals transitioned to more focused states from less focused states compared to the reverse. Patterns of transition were also highly systematic, as many specific transitions were more or less likely to occur than chance.

Hidden Markov states. Three hidden states were identified based on the optimal hidden Markov-chain model, describing the hidden response generation processes underlying the probe rating sequences. Additional hidden states did not greatly reduce the BIC of the model compared to the threestate solution (see Figure 4c). The three hidden state solution also recreated the empirically observed probe rating transition probabilities better than two hidden states. Correlations between empirically observed transition probabilities and simulated transition probabilities for 5000 sets of sequences were therefore larger on average for the three hidden state solution (mean $r=0.891, S D=0.009$, range $=.860-.921$ ), and the four state solution (mean $r=$ $0.905, S D=0.008$, range $=.876-.932)$, compared to the two state solution (mean $r=0.687, S D=0.012$, range $=.642-$ .737). Together with the BIC, these findings suggest that the three hidden state solution was an acceptable and parsimonious model of probe ratings. Table 3 provides initial probabilities, transition probabilities, and emission probabilities for two hidden state, three state, and four state Markov models. Figure 5 depicts a schematic representation illustrating steps in the empirical validation of hidden states.

Each of the three hidden states from the optimal Markov model are shown in Figure 4a as a circle chart in which the emission probabilities for probe ratings are depicted as proportions. Transition probabilities for hidden states are depicted in Figure 4b. All hidden states were highly likely $(\sim 95 \%)$ to transition to themselves. Hidden State 1 was characterized by a large probability of emitting probe ratings of $1(90.89 \%)$ and $2(6.21 \%)$. Hidden State 1 thus reflects a frequently occurring ( $66.15 \%$ of all probes belonged to this state based on the most probable hidden paths) and highly on-task state in which individuals infrequently report off-task probe ratings. When Hidden State 1 occurred, it had an average duration of 17.86 probes $(S D=10.64$, range $=1-$ 28 ). Based on the average number of trials between probes, the average duration of Hidden State 1 was therefore roughly 6.7 minutes. Hidden State 2 was characterized by the likelihood of emitting a range of probe ratings of $1(13.45 \%)$, $2(42.27 \%), 3(28.04 \%)$, and $4(11.95 \%)$. Hidden State 2 is 

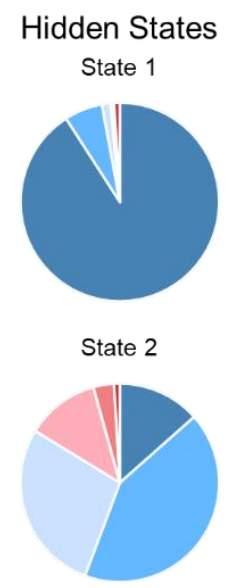

State 3

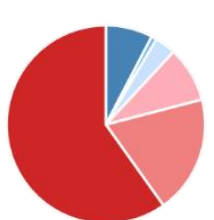

Simulated Probe Rating Sequences
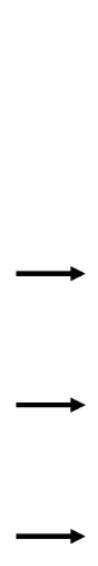

$$
N=500 \text { sequences }
$$

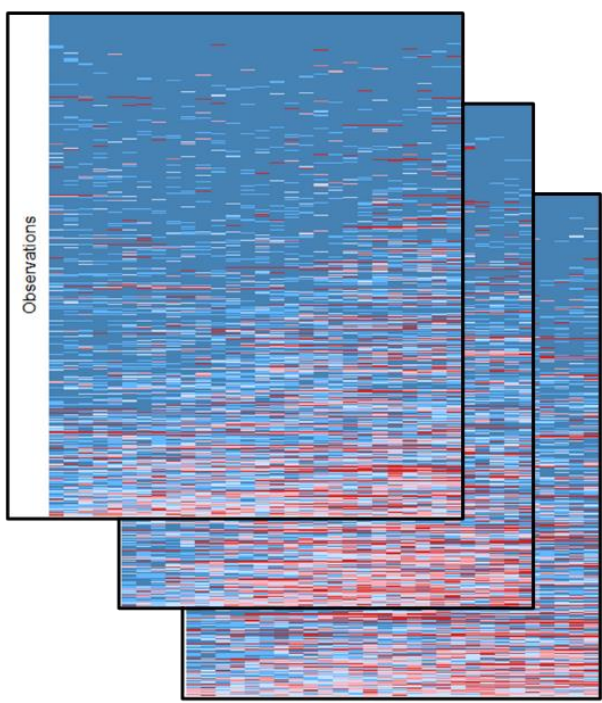

... 5000 simulations

\section{Simulated Transition Probabilities}

Transition To:

1. 2. 3. 4.5 .6 . 6 .

$\begin{array}{lllllll}1 . & 0.83 & 0.10 & 0.03 & 0.01 & 0.01 & 0.02\end{array}$

\begin{tabular}{l|llllll}
2. & 0.38 & 0.37 & 0.14 & 0.06 & 0.03 & 0.01
\end{tabular}

흔

\begin{tabular}{l|llllll}
3. & 0.19 & 0.26 & 0.27 & 0.16 & 0.08 & 0.04
\end{tabular}

0.02

\begin{tabular}{lllllll|l}
\hline 은 4. & 0.15 & 0.19 & 0.33 & 0.18 & 0.10 & 0.06 & 0.01
\end{tabular}

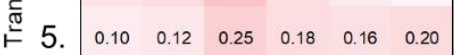

\begin{tabular}{lllllll|}
6. & 0.18 & 0.04 & 0.05 & 0.04 & 0.10 & 0.59 \\
\hline
\end{tabular}

\begin{tabular}{|llllll}
0.14 & 0.14 & 0.24 & 0.16 & 0.12 & 0.20
\end{tabular}

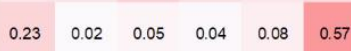

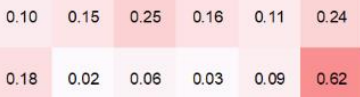

Figure 5: Schematic depicting empirical validation of hidden Markov models based on simulation of probe rating sequences. Hidden Markov states were identified from the observed probe rating sequences $(n=514)$. The three hidden state solution is depicted. 5000 sets of 500 probe rating sequences were simulated based on the initial probability of occurrence, transition probabilities, and emission probabilities of each hidden state solution. The transition probabilities of simulated probe ratings were then compared to the transition probabilities of observed probe ratings to evaluate how well each hidden state solution (e.g., 2 vs. 3 hidden states) recreates the transitions in the observed probe rating sequence data.
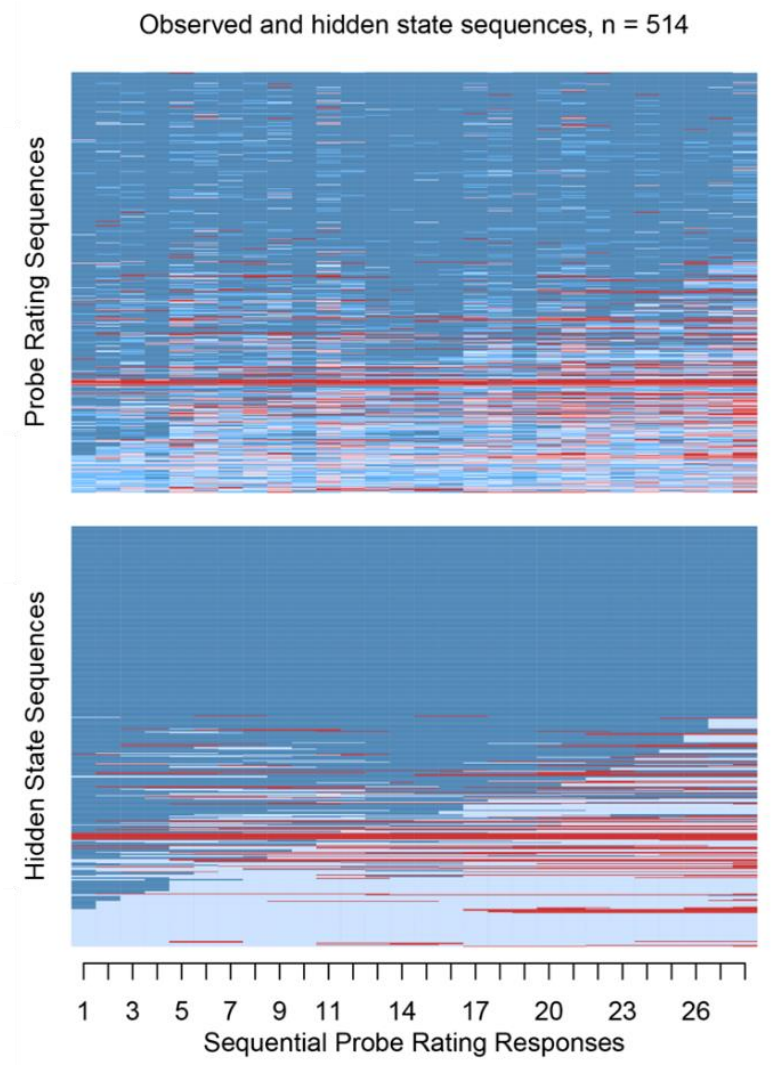

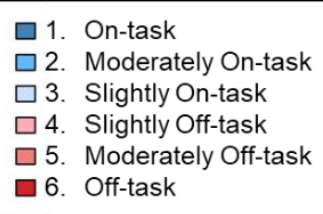

$\square$ State 1

$\square$ State 2

$\square$ State 3
Figure 6: Observed sequences of 28 probe ratings (rated from 1 "on-task" to 6 "off-task") during the SART are depicted on rows for each individual $(n=$ 514) above their corresponding hidden state sequences that illustrate the most probable hidden Markov paths underlying the probe rating responses. Sequences for individuals are sorted according to multidimensional scaling of hidden state sequence dissimilarities in order to group sequences on rows according to their similarity. 
Table 4: Model estimates of hidden state-related behavioral performance

\begin{tabular}{lcc}
\hline Parameter & Non-Target Accuracy & ICV \\
\hline Fixed Effects & & \\
$\quad$ Intercept & $96.535(0.308)^{* * *}$ & $0.218(0.005)^{* * *}$ \\
$\quad$ State 2 & $-0.961(0.328)^{* *}$ & $0.031(0.005)^{* * *}$ \\
$\quad$ State 3 & $-5.812(0.490)^{* * *}$ & $0.071(0.008)^{* * *}$ \\
Random Effects & & \\
$\quad$ Intercept variance & $39.352(2.715)$ & $0.012(0.001)$ \\
$\quad$ Residual variance & $111.890(1.343)$ & $0.025(0.001)$ \\
BIC & 109962 & -9464 \\
\# Observations & 14392 & 12397 \\
Subjects $(N)$ & 514 & 514 \\
\hline
\end{tabular}

Note: Maximum likelihood estimates are reported for mixed effects models of non-target accuracy (\% correct) and reaction time ICV calculated from the trials preceding each hidden Markov state. The model intercept reflects the estimated means for Hidden State 1, and the estimated difference is given for State 2 and State 3 . The number of subjects $(N)$ and observations contributing to the analyses are provided. Standard errors are reported in parentheses. $* * p$ $<.01, * * * p<.001$.

the second most common state $(25.44 \%$ of all probes) in which individuals are likely to report on-task probe ratings and accompanying mild to moderate attentional fluctuations. Hidden State 2 had an average duration of 12.80 probes ( $S D$ $=8.90$, range $=2-28)$. Hidden State 3 was characterized by high likelihood of emitting probe ratings of $5(19.21 \%)$ and 6 $(59.84 \%)$. Finally, Hidden State 3 was the least frequent state ( $8.40 \%$ of all probes) and had an average duration of 9.88 probes $(S D=8.32$, range $=1-28)$. Within individuals, Hidden State 1 was present among $88.13 \%$ of individuals' probe rating sequences, whereas Hidden State 2 was in $47.67 \%$ of sequences, and Hidden State 3 in $19.65 \%$ of sequences. Individuals on average experienced 1.55 ( $S D=$ $0.65,95 \%$ CI $[1.499,1.610])$ unique hidden states and had $0.83(S D=1.12,95 \%$ CI $[0.738,0.931])$ transitions between distinct occurrences of hidden states.

Figure 6 depicts the most probable hidden paths for each individual compared with the observed probe ratings. Rows are sorted according to multidimensional scaling of hidden state sequence dissimilarities based on optimal matching of edit distances in order to visually group individuals according to similarities in their sequence time series (Helske \& Helske, 2019). Visual inspection of the most probable hidden paths suggested a clear time-ordered decrease in the overall prevalence of Hidden State 1 and corresponding increase in the frequency of Hidden States 2 and 3. We statistically examined these changes using generalized linear mixed effects models to fit a cumulative logit proportionalodds model to the categorical hidden state data. Probe number was included as a fixed effect to estimate overall linear changes in the odds of state occurrences over time. Random participant intercepts were included, and parameters were estimated using maximum likelihood estimation based on adaptive quadrature. Accordingly, the odds of occurrence of Hidden State 1 (intercept $\log b=4.321,95 \%$ CI [3.798, 4.843]), and the cumulative odds of Hidden State 1 and 2 (intercept $\log b=8.383,95 \%$ CI [7.832, 8.933]), were reduced over the course of the task $(\log b=-0.110, p<.001$, $95 \%$ CI [-0.117, -0.102]). Individuals were therefore 0.896 times less likely to be in Hidden State 1 for each additional probe that was presented over time. This indicates an overall reduction in the proportion of Hidden State 1 over time and corresponding increases in Hidden States 2 and 3.

Hidden states were also behaviorally relevant. We examined differences in behavioral performance (non-target accuracy and reaction time ICV) for trials preceding each instance of a hidden state. Hidden states were treated as categorical predictors. Parameters from these models are reported in Table 4. For non-target accuracy, Hidden State 2 had significantly worse non-target accuracy than Hidden State 1 by $-0.961 \%$ ( $p=.004,95 \%$ CI [-1.607, -0.316$])$. Hidden State 3 had worse non-target accuracy than Hidden State 1 by $-5.812 \%(p<.001,95 \%$ CI [-6.777, -4.848]). For ICV, Hidden State 2 had significantly greater ICV than Hidden State 1 by 0.043 ( $p<.001,95 \%$ CI [0.031, 0.055]). Hidden State 3 had greater ICV than Hidden State 1 by 0.099 ( $p<$ $.001,95 \%$ CI $[0.081,0.117])$. These findings demonstrate that accuracy was worse, and variability was greater, in the moments preceding probe ratings classified as belonging to Hidden State 2 and Hidden State 3, compared to Hidden 

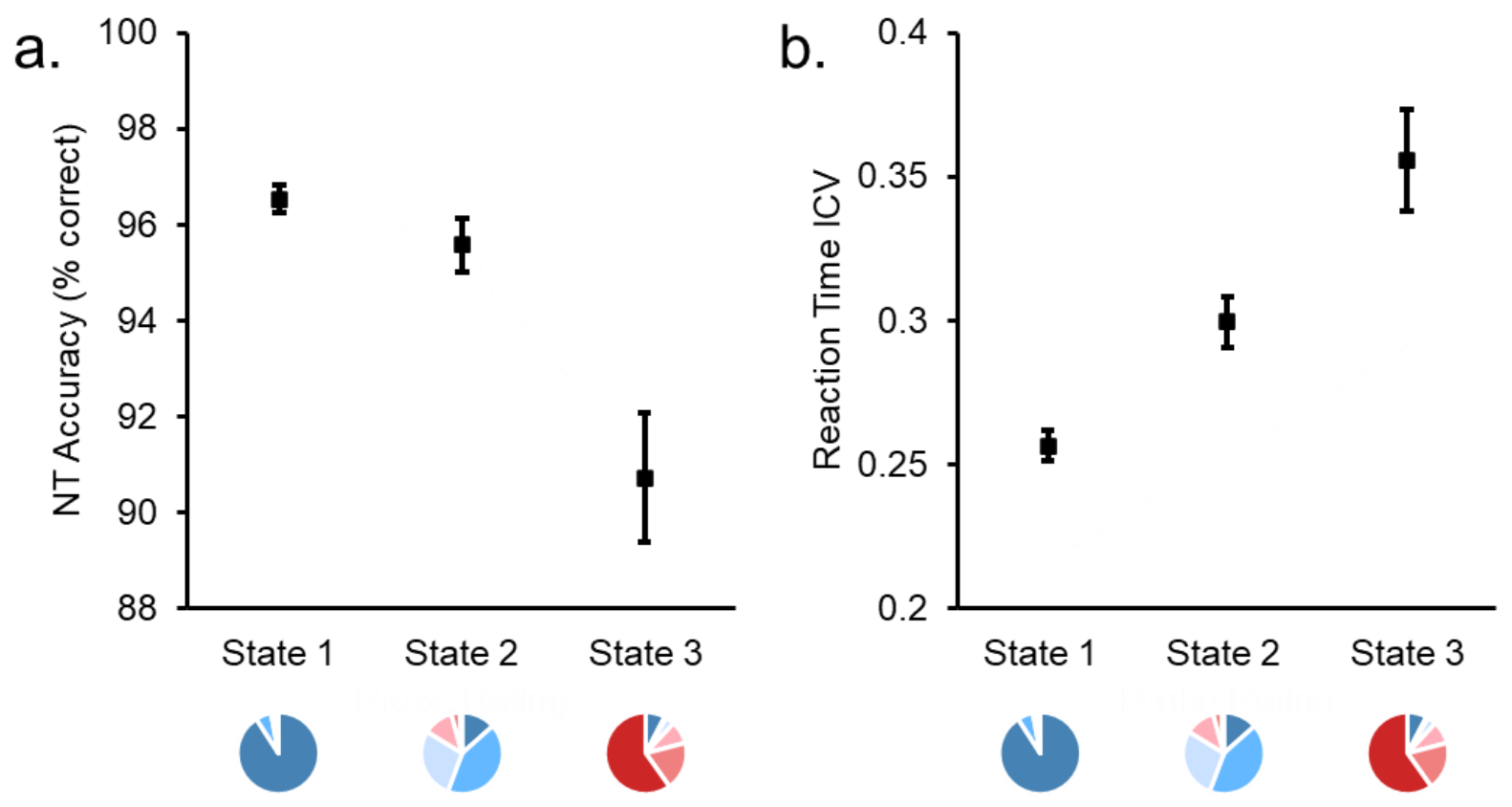

Figure 7: Mixed effects model estimated means for (a.) non-target accuracy (\% trials correct) and (b.) reaction time ICV for trials preceding each hidden state. Error bars are standard errors of the estimate.

State 1. Hidden State 3, however, was associated with even greater accuracy deficits and increases in variability than those associated with Hidden State 2 (see Figure 7).

\section{Replication in Two Independent Datasets}

Given that analyses of transition probabilities and hidden Markov models are data-driven and necessarily more exploratory than other analyses, we examined whether patterns of transitions and identification of hidden Markov states generalized to two independent data sets. We therefore conducted secondary analysis of deidentified participant data that were acquired via the Open Science Framework provided by Goller et al. (2020; https://osf.io/shxb6/) and by Wiradhany et al. (2019; https://osf.io/nkdw5/). The results of these analyses are provided in Supplementary Materials. In both cases, three hidden Markov states were identified as the optimal model of probe ratings in the SART (Goller et al., 2020) and a working memory change detection task (Wiradhany et al., 2019). The three hidden state solutions and the most probable hidden paths for individuals are summarized for both studies in Figure 8.

\section{Discussion}

Task-embedded experience sampling has been utilized for more than a decade to track and assess states of mind wandering and task-unrelated thought during cognitive task performance (Smallwood \& Schooler, 2006, 2015). The results of the present study suggest that attentional lapses assessed by experiential reports of the degree of one's current attentional focus are more varied than can be explained by discrete episodes of task engagement (i.e., ontask) and mind wandering (i.e., off-task) alone. Instead, participants as a whole used a large range of response options in categorizing their current attentional state, and, as observed in prior studies (i.e., Laflamme et al., 2018), the continuum of probe ratings were linearly associated with reaction time variability in the moments preceding probes. Individuals can therefore seemingly distinguish among a continuity of behaviorally relevant fluctuations in their current degree of attentional engagement, arguing for a continuum of attentional states contributing to probe-rating responses and behavioral performance in tasks requiring sustained attention.

There was, however, some indication that experience sampling probes can also capture more discrete episodes of complete disengagement as reflected in the non-linear association between probe ratings and preceding non-target accuracy. Highly off-task ratings (i.e., probe ratings of 6) were more strongly associated with gross behavioral disengagement than less off-task ratings, as indicated by poorer non-target accuracy in moments preceding these offtask probe ratings. Individuals were therefore most likely to fail at the most basic task instructions - correctly responding to non-targets - in the moments preceding highly off-task reports. This non-linear association implies something unique, and perhaps more categorical, about moments reported to be highly on-task or highly off-task that are distinguished from the gradation of ratings between them. In contrast, linear associations with ICV demonstrated that 

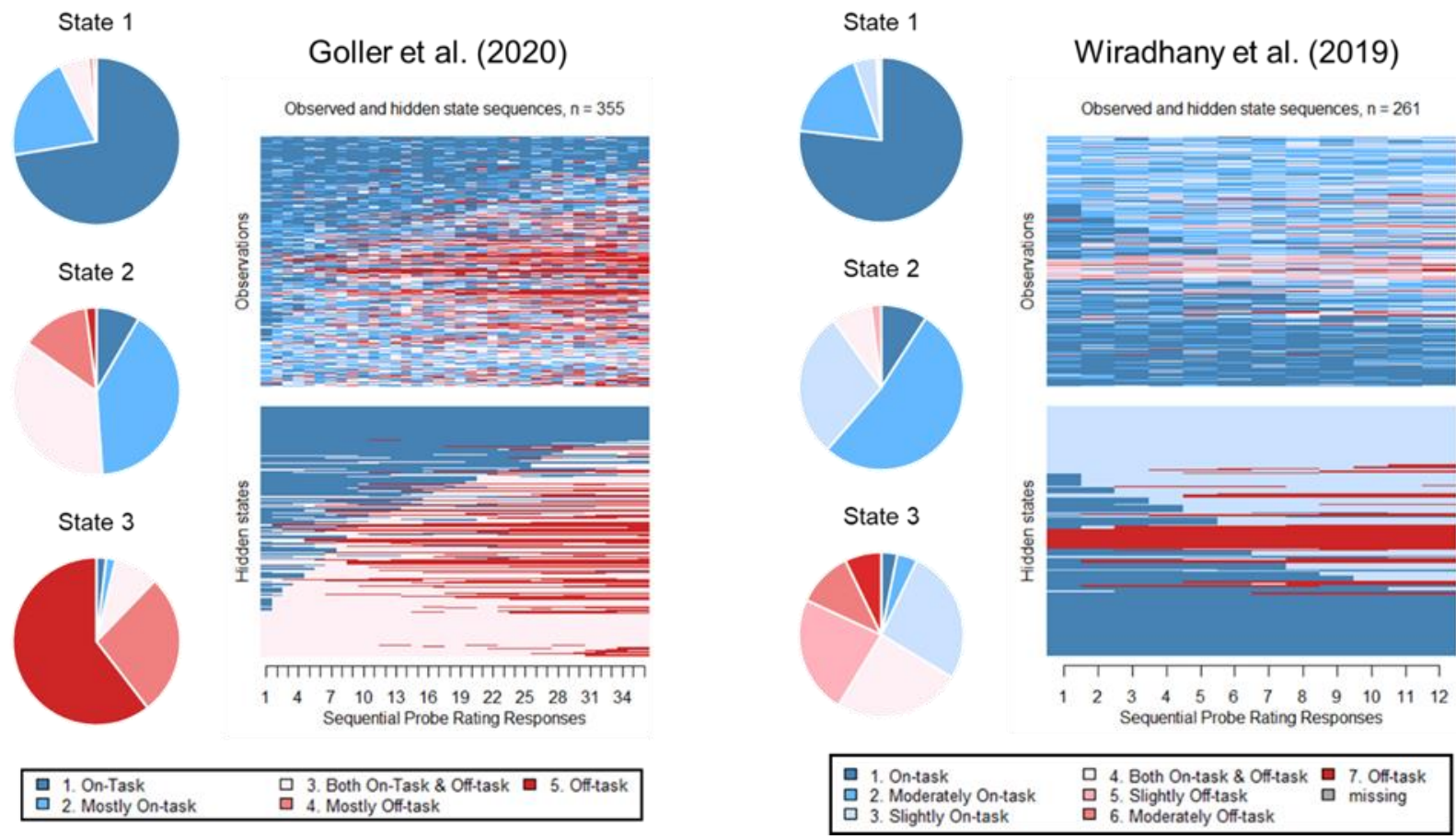

Figure 8: Three hidden Markov states are depicted from the optimal models of probe rating sequences from studies by Goller et al. (2020) and Wiradhany et al. (2019). Each state is depicted as a circle chart with emission probabilities given as proportions of each state. The observed sequences are also depicted on rows for individuals alongside their corresponding hidden state sequences. Sequences for individuals are sorted according to their hidden state sequence dissimilarities in order to group sequences on rows according to their similarity.

linear gradations in ratings also reflect behaviorally relevant attentional fluctuations. These distinct patterns of linear and non-linear associations illustrate the value of evaluating nonlinear associations to better understand the measurement continua of constructs (Tay \& Jebb, 2018). Together, the present findings suggest that attentional fluctuations measured using a Likert-like scale reflect a mixture of several response-generating processes: discrete states of focus and disengagement, and the graded continuum of attentional fluctuations between them.

Markov-chain modeling of probe rating sequences provided further support for this supposition. Highly on-task ratings (i.e., ratings of 1) and highly off-task ratings (6) were more than twice as likely (64-84\%) to linger in time from probe to probe compared to other probe ratings. In contrast, probe ratings of 2,3,4, and 5, were more likely to fluctuate between other ratings. Probe ratings of 1 and 6 thus appear qualitatively distinct from ratings of 2, 3, 4, and 5, based on their transition probabilities. These patterns accord well with three hidden Markov states identified from the probe rating sequences that appear to represent distinct responsegenerating processes. The first hidden state corresponds to a task-focused state in which individuals were generally ontask, but infrequently reported transient attentional lapses that did not appear to disrupt their task-related focus in a lasting manner. The second hidden state corresponds to a more heterogeneous state contributing to a range of subtler attentional fluctuations across the continuum of probe rated responses. The third hidden state corresponds to a highly unfocused state with a higher probability of off-task reports. Finally, hidden states demonstrated similar associations with behavioral performance as probe ratings and are in line with the supposition that individuals can distinguish their current attentional states in degree and in kind.

Hidden attentional states identified from probe ratings were highly likely to persist over time according to patterns of transitions among states. Hidden states endured for roughly ten to twenty probes based on estimates of their average duration. This suggests that these hidden attentional states are more global than transient, and presumably exert their influence over hundreds of task trials. The first hidden state was also more temporally stable (i.e., longer in duration) compared to other hidden states. In part, this is in line with prior hidden Markov modeling incorporating reaction times (Bastian \& Sackur, 2013), which suggested that "on-task" periods of responding last longer than "off-task" periods. But hidden states identified from fluctuations in reaction times were considerably more volatile than those found herein, persisting for only 10 to 20 seconds at a time (Bastian \& Sackur, 2013). These inherent differences highlight the many attentional rhythms contributing to task performance that interact at multiple temporal scales (Shalev, Bauer, \& Nobre, 2019), which can be identified from distinct fluctuations in behavioral performance and subjective experience. 
The frequency and prevalence of mind wandering in everyday life and during cognitive tasks has been estimated to range between $30-50 \%$ of the time based on dichotomous probe-caught estimates (Kane et al., 2007, 2016, 2017; Killingsworth \& Gilbert, 2010). In line with a prior study that directly compared dichotomous to continuous proberatings in daily life (Seli et al., 2018a), the present findings suggest that the frequency of task-unrelated thought in goaldirected cognitive tasks such as the SART is likely inflated. The vast majority (87\%) of all 14393 probe ratings in the present study were rated lower than 4 , and highly off-task ratings (a rating of 6) occurred only on $6.09 \%$ of all probes. Hidden State 3, the hidden state most likely to contribute to episodes of task-unrelated thought, was present for only $8.40 \%$ of all probes. One would have to classify a large range of response options as occurrences of task-unrelated thought to be comparable with commonly observed frequencies reported from other studies using dichotomous approaches. This would seem inappropriate, as we provided evidence suggesting that not all probe ratings likely originate from the same response-generating process, given the highly skewed response distribution, linear and quadratic relations with behavior in the moments preceding probes, and distinct hidden Markov states that reflect unique and dynamic patterns of transitions between attentional states. As Seli et al. (2018a) note, attempting to estimate the true frequency of mind wandering using a single dichotomous criterion may not be very psychologically informative.

Alongside examination of the frequency of probe ratings, transitions among probe ratings may yield important insights into the temporal structure of task-related cognition.

Transition probabilities indicated that individuals were highly likely to remain in the same probe-rated attentional state over time, but also worked to regulate their attention by returning focus to more on-task states following some reported lapse. This is consistent with the general capacity of individuals to strategically regulate their attention and mind wandering in response to dynamic situational demands in the cognitive task context (Seli et al., 2018b). Alternatively, task-unrelated thought and attentional disengagement may linger and reoccur over time in individuals who have trouble concentrating. Importantly, we also observed time-ordered decreases in the prevalence of Hidden State 1 over the course of task performance, and corresponding increases in Hidden State 2 and 3. Theories attempting to explain associations between mind wandering and sustained attention (i.e., Thomson, Besner, \& Smilek, 2015) must account for not only increased frequency of off-task reports with time-ontask but possible changes in the temporal dependencies between probe ratings and their underlying hidden states.

Importantly, hidden states and patterns of transition probabilities appeared to generalize to other samples of individuals and different cognitive tasks. Goller et al. (2020) had 356 undergraduate participants complete the SART, in which experience sampling probes first assessed the content of individuals' thoughts and then the degree of their taskrelated focus (see Supplementary Materials). Three hidden states better explained the time series sequence of attentional probe ratings than two hidden states and had similar patterns of emission probabilities as those identified in the present study. Importantly, the content of individuals' thoughts in the study by Goller et al. (2020) were also distinguished based on the corresponding hidden states of attentional probes. Specifically, Hidden State 1 and 2 were mainly associated with task-related thoughts pertaining to the current task or evaluations of their performance. Hidden State 3, on the other hand, primarily accompanied task-unrelated thoughts. Episodes of task-unrelated thought are thus likely indexed by highly off-task probe ratings in contrast to more gradual attentional fluctuations and task-related interference captured by the broader range of ratings.

Wiradhany et al. (2019) had 261 participants complete a working memory task in which experience sampling probes assessed the degree of their task-related focus following blocks of trials (see Supplementary Materials). Again, three hidden states explained the sequence of probe ratings better than two hidden states. While the first and second hidden state strongly resembled those identified in the present study, the third hidden state did not contain as high a proportion of off-task reports as in the other studies. Together, these independent studies importantly demonstrate the identification of similar hidden attentional states in other task contexts and samples of participants. It should be noted that participants in the present study tended to report being more on-task than those in the study by Goller et al. (2020). While Hidden State 3 occurred more frequently $(\sim 17 \%)$ in the two independent studies, the proportion of highly off-task probes was also higher (12\%) in the study by Goller et al. (2020) than in the present study. It is possible that the inclusion of thought probes alongside attentional probe ratings by Goller et al. (2020) may have increased the prevalence with which individuals rate their attention as off-task. On the other hand, off-task reports may be low in the present study because of different task features such as probe presentation rate (cf. Schubert, Frischkorn, \& Rummel, 2019; Seli, Carriere, Levene, \& Smilek, 2013), and participants were military service members who may have different reporting biases, interpretation of task instructions, or may have tried harder to concentrate (Kane et al., 2017), than college undergraduates. Studies should continue to examine patterns in the time series sequence of probe ratings to elucidate the factors that contribute to the prevalence of mind wandering and the psychological dynamics of these experiences in different cognitive task contexts (e.g., Zanesco, 2020). 
The acknowledged conceptual heterogeneity surrounding definitions of mind wandering (Seli et al., 2018c) mirrors considerable methodological variance in experience sampling probe questions and response options between studies (Weinstein, 2018). Methodological variability may be useful, however, in that any one approach may not adequately assess the variety of experiences that arise in individuals during ongoing task performance. But the present findings also suggest that it may not be possible to assess specific aspects of individuals' attentional state without conflating some experiential qualities with others. It would be valuable for researchers to continue to evaluate the construct validity of experience sampling measures of mind wandering, with attention to the construct continuum assessed by probe ratings (Tay \& Jebb, 2018). Using one of the more common mind wandering probe response options (Weinstein, 2018), we found that individuals likely draw on a range of distinct experiences to classify their focus of attention on a continuum from completely on to completely off-task.

Future studies will need to consider these issues carefully. Failure to account for the distinct attentional processes underlying probe ratings could lead to inaccurate conclusions about individuals' behavior or experience. In some cases, for example, only highly off-task probe reports might be associated with certain study outcomes because those more likely reflect episodes of engrossing task-unrelated thought. Possible methodological solutions may be to better distinguish these experiences by collecting multidimensional information with probes assessing the many attentional experiences contributing to task performance, and by refining experience sampling questions to minimize response biases and target internal experiences with greater specificity. Given that internal experiences such as mind wandering are hard to report on with precision (Meier, 2018), such an approach may require training individuals to report on details of their phenomenal experience to a greater degree than is commonly done with naïve study participants (cf., Abdoun, Zorn, Poletti, Fucci, \& Lutz, 2019). Methods that can better account for the temporal dynamics of probe ratings might also help researchers address these questions further. In addition to other sequence analytic methods (Zanesco, 2020), Markov-chain modeling of categorical time series provides one effective methodology for describing and analyzing the dynamics of experience sampling probe sequences.

Our findings are broadly in line with theories of attentional fluctuations in goal-directed cognitive tasks that suggest attention fluctuates to varying degrees between optimal periods of engagement and stability, and periods of instability, degraded attentional control, and difficulty in perceptual processing (Adam, Mance, Fukuda, \& Vogel, 2015; deBettencourt, Keen, Awh \& Vogel, 2019; Esterman,
Noonan, Rosenberg, \& DeGutis, 2013; Esterman, Rosenberg \& Noonan, 2014; Rothlein, DeGutis, \& Esterman, 2018). The constellation of causes for such instability remains to be fully characterized, but includes intrinsic fluctuations in arousal, cognitive control, and perceptual processing, and episodes of task-related interference and task-unrelated thought (Esterman \& Rothlein, 2019; Shalev et al., 2019; Smallwood \& Schooler, 2015). Of note, individuals experience and appear able to report on these fluctuations, and the frequency and pattern with which individuals report on their task-related attention suggests several meaningful sources of variation contribute to experience sampling behavior.

\section{Acknowledgements}

We thank Nicholas Ramos, Merissa Goolsarran, Elliot TangSmith, Laura MacKinnon, Keith Chichester, Emily

Schwartz, and Lindsey Slavin for their assistance with data collection. We further thank Haley Goller, Jonathan Banks, and Matt Meier, and Wisnu Wiradhany, Marieke van Vugt, and Mark Nieuwenstein, for sharing their deidentified data online. This research was supported by Department of Army grant \#W81XWH-12-2-0051 and Henry Jackson Foundation Grant \#HU0001-15-2-0003 with subaward \#3479 to APJ.

\section{Data Availability Statement}

The probe rating data that support the findings of this study are available on the Open Science Framework at https://osf.io/rf9jh/

\section{References}

Abdoun, O., Zorn, J., Poletti, S., Fucci, E., \& Lutz, A. (2019). Training novice practitioners to reliably report their meditation experience using shared phenomenological dimensions. Consciousness and Cognition, 68, 5772. https://doi.org/10.1016/j.concog.2019.01.004

Adam, K. C. S., Mance, I., Fukuda, K., \& Vogel, E. K. (2015). The contribution of attentional lapses to individual differences in visual working memory capacity. Journal of Cognitive Neuroscience, 27(8), 1601-1616. https://doi.org/10.1162/jocn_a_00811

deBettencourt, M. T., Keene, P. A., Awh, E., \& Vogel, E. K. (2019). Realtime triggering reveals concurrent lapses of attention and working memory. Nature Human Behavior, 3, 808-816. https://doi.org/10.1038/s41562-0190606-6

Christoff, K., Irving, Z. C., Fox, K. C. R., Spreng, N., \& Andrews-Hanna, J. R. (2016). Mind-wandering as spontaneous thought: a dynamic framework. Nature Reviews Neuroscience, 17, 718-731.

https://doi.org/10.1038/nrn.2016.113

Esterman, M., Noonan, S. K., Rosenberg, M., \& DeGutis, J. (2013). In the zone or zoning out? Tracking behavioral and neural fluctuations during sustained attention. Cerebral Cortex, 23, 2712-2723.

https://doi.org/10.1093/cercor/bhs261 
Esterman, M., Rosenberg, M. D., \& Noonan, S. K. (2014). Intrinsic fluctuations in sustained attention and distractor processing. Journal of Neuroscience, 34(5), 1724-1730. https://doi.org/10.1523/JNEUROSCI.2658-13.2014

Esterman, M., \& Rothlein, D. (2019). Models of sustained attention. Current Opinion in Psychology, 29, 174-180. https://doi.org/10.1016/j.copsyc.2019.03.005

Gabadinho, A., Ritschard, G., Müller, N. S., \& Studer, M. (2011). Analyzing and visualizing state sequences in $\mathrm{R}$ with TraMineR. Journal of Statistical Software 40(4), 1-37. https://doi.org/10.18637/jss.v040.i04

Goller, H., Banks, J. B., \& Meier, M. E. (2020). An individual differences investigation of the relations among life event stress, working memory capacity, and mind wandering: A preregistered replication-extension study. Memory \& Cognition. https://doi.org/10.3758/s13421-020-01014-8

Hawkins, G. E., Mittner, M., Forstmann, B. U., \& Heathcote, A. (2019). Modeling distracted performance. Cognitive Psychology, 112, 48-80.

Helske, S, \& Helske, J (2019). Mixture Hidden Markov Models for Sequence Data: The seqHMM Package in R. Journal of Statistical Software, 88(3), 1-32. https://doi.org/10.18637/jss.v088.i03

Irving, Z. C. (2016). Mind-wandering is unguided attention: Accounting for the "purposeful" wanderer. Philosophical Studies, 173(2), 547-571. https://doi.org/10.1007/s11098-015-0506-1

Irving, Z. C., \& Thompson, E. (2018). The philosophy of mind-wandering. In K. C. R. Fox \& K. Christoff (Eds.), The Oxford Handbook of Spontaneous Thought: Mind-wandering, Creativity, and Dreaming (pp. 8796). New York, NY: Oxford University Press. https://doi.org/10.1093/oxfordhb/9780190464745.013.19

Kane, M. J., Brown, L. H., McVay, J. C., Silvia, P. J., Myin-Germeys, I., \& Kwapil, T. R. (2007). For whom the mind wanders, and when: an experience-sampling study of working memory and executive control in daily life. Psychological Science, 18(7), 614-621.

https://doi.org/10.1111/j.1467-9280.2007.01948.x

Kane, M. J., Gross, G. M., Chun, C. A., Smeekens, B. A., Meier, M. E., Silvia, P. J., \& Kwapil, T. R. (2017). For whom the mind wanders, and when, varies across laboratory and daily-life settings. Psychological Science, 28(9), 1271-1289. https://doi.org/10.1177/0956797617706086

Kane, M. J., Meier, M. E., Smeekens, B. A., Gross, G. M., Chun, C. A., Silvia, P. J., \& Kwapil, T. R. (2016). Individual differences in the executive control of attention, memory, and thought, and their associations with schizotypy. Journal of Experimental Psychology: General, 145(8), $1017-$ 1048. https://doi.org/10.1037/xge0000184

Killingsworth, M. A., \& Gilbert, D. T. (2010). A wandering mind is an unhappy mind. Science, 330(6006), 932. https://doi.org/10.1126/science.1192439

Krimsky, M., Forster, D. E., Llabre, M. M., \& Jha, A. P. (2017). The influence of time on task on mind wandering and visual working memory. Cognition, 169, 84-90. https://doi.org/10.1016/j.cognition.2017.08.006

Krosnick, J. A. (1999). Survey research. Annual Review of Psychology, 50, 537-567. https://doi.org/10.1146/annurev.psych.50.1.537

Laflamme, P., Seli, P., \& Smilek, D. (2018). Validating a visual version of the metronome response task. Behavior Research Methods, 50, 1503-1514. https://doi.org/10.3758/s13428-018-1020-0
Meier, M. E. (2018). Can research participants comment authoritatively on the validity of their self-reports of mind-wandering and task-engagement? A replication and extension of Seli, Jonker, Cheyne, Cortes, and Smilek (2015). Journal of Experimental Psychology: Human Perception and Performance, 44(10), 1567-1585. https://doi.org/ doi:10.1037/xhp0000556

Randall, J. G., Oswald, F. L., \& Beier, M. E. (2014). Mind-wandering, cognition, and performance: A theory-driven meta-analysis of attention regulation. Psychological Bulletin, 140(6), 1411-1431. https://doi.org/10.1037/a0037428

Robertson, I. H., Manly, T., Andrade, J., Baddeley, B. T., \& Yiend, J. (1997). 'Oops!': Performance correlations of everyday attentional failures in traumatic brain injured and normal subjects. Neuropsychologia, 35(6), 747 758. https://doi.org/10.1016/S0028-3932(97)00015-8

Robison, M. K., Miller, A. L., \& Unsworth, N. (2019). Examining the effects of probe frequency, response options, and framing within the thought-probe method. Behavior Research Methods. https://doi.org/10.3758/s13428-019-01212-6

Rothlein, D., DeGutis, J., \& Esterman, M. (2018). Attentional fluctuations influence the neural fidelity and connectivity of representations. Journal of Cognitive Neuroscience, 30(9), 1209-1228.

https://doi.org/10.1162/jocn_a_01306

Schubert, A. L., Frischkorn, G. T., \& Rummel, J. (2019). The validity of the online thought-probing procedure of mind wandering is not threatened by variations of probe rate and probe framing. Psychological Research. https://doi.org/10.1007/s00426-019-01194-2

Seli, P., Beaty, R. E., Cheyne, J. A., Smilek, D., Oakman, J., \& Schacter, D L. (2018a). How pervasive is mind wandering, really? Consciousness and Cognition, 66, 74-78. https://doi.org/10.1016/j.concog.2018.10.002

Seli, P., Carriere, J. S., Levene, M., \& Smilek, D. (2013). How few and far between? Examining the effects of probe rate on self-reported mind wandering. Frontiers in Psychology, 4:430. https://doi.org/10.3389/fpsyg.2013.00430

Seli, P., Carriere, J. S., Wammes, J. D., Risko, E. F., Schacter, D. L., \& Smilek, D. (2018b). On the clock: evidence for the rapid and strategic modulation of mind wandering. Psychological Science, 29(8), 1247-1256. https://doi.org/10.1177/0956797618761039

Seli, P., Kane, M. J., Smallwood, J., Schacter, D. L., Maillet, D., Schooler, J. W., \& Smilek, D. (2018c). Mind-wandering as a natural kind: A family resemblances view. Trends in Cognitive Sciences, 22(6), 479-490. https://doi.org/10.1016/j.tics.2018.03.010

Shalev, N., Bauer, A. R., \& Nobre, A. C. (2019). The tempos of performance. Current Opinion in Psychology, 29, 254-260. https://doi.org/10.1016/j.copsyc.2019.06.003

Shannon, C. E. (1948). A mathematical theory of communication. Bell System Technical Journal, 27(3), 379-423.

Smallwood, J., \& Schooler, J. W. (2006). The restless mind. Psychological Bulletin, 132(6), 946-958. https://doi.org/10.1037/0033-2909.132.6.946

Smallwood, J., \& Schooler, J. W. (2015). The science of mind wandering: Empirically navigating the stream of consciousness. Annual Review of Psychology, 66, 487-518. https://doi.org/10.1146/annurev-psych-010814015331 
Stanislaw, H., \& Todorov, N. (1999). Calculation of signal detection theory measures. Behavior Research Methods, Instruments, \& Computers, 31(1), 137-149. https://doi.org/10.3758/BF03207704

Tay, L., \& Jebb, A. T. (2018). Establishing construct continua in construct validation: The process of continuum specification. Advances in Methods and Practices in Psychological Science, 1(3), 375-388. https://doi.org/10.1177/2515245918775707

Thomson, D. R., Besner, D., \& Smilek, D. (2015). A resource-control account of sustained attention: evidence from mind-wandering and vigilance paradigms. Perspectives on Psychological Science, 10(1), 82-96. https://doi.org/10.1177/1745691614556681

Thomson, D. R., Seli, P., Besner, D., \& Smilek, D. (2014). On the link between mind wandering and task performance over time. Consciousness and Cognition, 27, 14-26. https://doi.org/10.1016/j.concog.2014.04.001

Visser, I., Raijmakers, M. E. J., \& Molenaar, P. C. M. (2002). Fitting hidden Markov models to psychological data. Scientific Programming, 10, 185-199. https://doi.org/10.1155/2002/874560

Weinstein, Y. (2018). Mind-wandering, how do I measure thee with probes? Let me count the ways. Behavior Research Methods, 50, 642-661. https://doi.org/10.3758/s13428-017-0891-9

Wiradhany, W., van Vugt, M. K., \& Nieuwenstein, M. R. (2019). Media multitasking, mind-wandering, and distractibility: A large-scale study. Attention, Perception, \& Psychophysics. https://doi.org/10.3758/s13414019-01842-0

Zanesco, A. P. (2020). Quantifying streams of thought during cognitive task performance using sequence analysis. Behavior Research Methods. https://doi.org/10.3758/s13428-020-01416-1 


\section{Supplementary Materials}

Deidentified participant data were acquired via the Open Science Framework provided by Goller et al. (2020; https://osf.io/shxb6/) and Wiradhany et al. (2019; https://osf.io/nkdw5/). Secondary analysis of these data used the same participant exclusions and outlier treatment as originally reported by Goller et al. (2020) and Wiradhany et al. (2019).

\section{Participants}

Goller et al. (2020) assessed 371 undergraduates at Western Carolina University. Complete details regarding participant recruitment and demographic characteristics are reported elsewhere (Goller et al., 2020). After accounting for performance outliers and exclusions, 356 participants were included in analyses of SART performance and experience sampling probes. One additional participant was removed from analyses of attentional probe ratings because of missing data, leaving 355 participants with complete ratings of the degree of their task-related focus. All participants provided informed consent in accordance with the Institutional Review Board of Western Carolina University.

Wiradhany et al. (2019) assessed 275 participants. Complete details regarding participant recruitment and demographic characteristics are reported elsewhere (Wiradhany et al., 2019). After accounting for performance outliers and exclusions, 261 participants were included in analyses of working memory task performance and experience sampling probes. Data from 107 participants were collected in person in a laboratory setting, whereas the remaining 154 participants used their own computers to complete the assessment as part of an assignment for an undergraduate research practicum course. All participants provided informed consent in accordance with the Ethics Committee of the Psychology Department at the University of Groningen.

\section{Procedure}

The complete description of study procedures and the order of cognitive task administration is described by Goller et al. (2020) and Wiradhany et al. (2019). Goller et al. (2020) administered the SART to participants as part of a battery of cognitive behavioral tasks. The SART contained 540 total trials in which participants responded to non-target stimuli (words relating to animals) by pressing the spacebar. Participants withheld their response to target stimuli (words relating to vegetables). 36 pairs of experience sampling questions were randomly presented during task performance. The first experience sampling question asked about the content of participant's thought at the moment of presentation (i.e., "What were you just thinking about?"). There were six categorical response options that included: 1) task-related thoughts pertaining to the current task; 2) positive task-related evaluative thoughts; 3) negative taskrelated evaluative thoughts; 4) neutral task-unrelated thoughts; 5) positive task-unrelated thoughts; and 6) negative task-unrelated thoughts. Importantly, a second probe question immediately followed the first, which asked how "on or off-task" they were according to a 5-point Likert scale (from 1 "completely on-task" to 5 "completely off-task").

Wiradhany et al. (2019) administered a working memory change-detection task. Participants were asked to memorize the orientiation of two, four, or six target objects and ignore distracter objects, and report on any change in orientation of the targets following a delay. After blocks of 16 trials each, experience sampling probes were presented that asked participants to rate the degree of their task-related focus from on to off-task according to a 7-point Likert scale (from 1 "on-task" to 7 "off-task"). A second probe question was subsequently presented asking participants to report the degree to which they were aware of their focus of attention according to a 7-point Likert scale (from 1 "aware" to 7 "unaware"). There were 12 experience sampling probes in total, and probes were not presented after the last block of 8 trials.

\section{Analysis}

The frequency distribution of attentional probe ratings ("on-task" to "off-task"), their Markov-chain transition probabilities, and hidden Markov states were characterized for each set of data for studies by Goller et al. (2020) and Wiradhany et al. (2019). Methods for these analyses are the same as those reported in the Methods section of the main manuscript. Some probe ratings were missing for individuals in the study by Wiradhany et al. (2019). In these cases, missing data were ignored in estimating transition probabilities and hidden Markov models.

\section{Results}

\section{Secondary Analysis of Goller et al. (2020)}

Frequency distribution of attentional probe ratings. Supplementary Figure 1a depicts the sequences of attentional probe ratings (from 1 "on-task" to 5 "off-task") during the SART for all individuals. The distribution of all 12,780 attentional probe ratings (see Supplementary Figure 1b) was strongly positively skewed with $29.08 \%$ of all attentional probe ratings rated equal to 1 (on-task). $70.92 \%$ of all probes were rated greater than $1.23 .58 \%$ of probes were rated 4 or greater, and $12.05 \%$ of all probes were rated 5 (off-task). 
Supplementary Table 1: Distribution of probe ratings for Goller et al. (2020) and Wiradhany et al. (2019)

\begin{tabular}{lccccccc}
\hline & \multicolumn{7}{c}{ Probe Rating } \\
\cline { 2 - 8 } & 1 & 2 & 3 & 4 & 5 & 6 & 7 \\
\hline Goller et al. (2020) & 0.291 & 0.267 & 0.206 & 0.115 & 0.121 & & \\
Wiradhany et al. (2019) & 0.343 & 0.304 & 0.188 & 0.078 & 0.049 & 0.021 & 0.016 \\
\hline
\end{tabular}

Note: The proportion of all probe ratings classified as a particular probe rating response (1 "on-task" to 5 "off-task" or 1 "on-task" to 7 "off-task") are provided for studies by Goller et al. (2020) and Wiradhany et al. (2019).

Participants on average reported 4.07 ( $S D=0.96,95 \%$ CI [3.973, 4.173], range $=1-5$ ) unique categories of attentional probe ratings. Participants, as a whole, used a range of responses to categorize their current attentional state. Individuals were less likely to report being off-task to some extent (i.e., a probe rating of 4 or 5) than they were on-task.

Transition Probabilities of attentional probe ratings. Markov-chain transition probabilities for attentional probe ratings revealed strong transition probabilities along the diagonal of the transition probability matrix. Supplementary Figure 1c depicts the transition probability matrix of all attentional probe ratings.

Participants on average had $17.41(S D=7.06,95 \%$ CI $[16.679,18.149])$ transitions between distinct probe ratings. Transitions were more likely to occur than chance (i.e., transition probabilities more extreme than $97.5 \%$ of 5,000 random permutations of probe rating sequences) for transitions between ratings of $1 \rightarrow 1,2 \rightarrow 2,3 \rightarrow 3,4 \rightarrow 4$, and $5 \rightarrow 5$. Transitions were also more likely than chance to occur for transitions between ratings of $5 \rightarrow 4$. Individuals were also likely $(62.9 \%)$ to remain in a highly focused state following a report of being on-task (i.e., a probe rating of 1), as transitions from ratings of $1 \rightarrow 3,1 \rightarrow 4$, and $1 \rightarrow 5$, occurred less frequently than expected by chance alone. After experiencing some degree of lapsing focus, however, individuals were less likely than chance to transition from ratings of $2 \rightarrow 1,3 \rightarrow 1,4 \rightarrow 1$, and $5 \rightarrow 1$. Following a complete attentional lapse, individuals were highly likely $(66.8 \%)$ to remain in that same off-task state (i.e., probe rating of 5) during the next probe question, and were less likely than chance to transition to more on-task states for transitions between ratings of $5 \rightarrow 1,5 \rightarrow 2$, and $5 \rightarrow 3$. Transitions were also less likely than chance to occur for transitions between ratings of $2 \rightarrow 4,2 \rightarrow 5,3 \rightarrow 2,3 \rightarrow 4,3$ $\rightarrow 5$, and $4 \rightarrow 2$. All other transitions were no more or less extreme compared to chance alone.

Hidden Markov states. Three hidden states were identified based on the optimal hidden Markov-chain model. Each of the three hidden states from the optimal Markov model are shown in Figure 2a as a circle chart in which the emission probabilities for probe ratings are depicted as proportions. Additional hidden states did not greatly reduce the BIC of the model compared to the three-state solution (see
Supplementary Figure 2c). The three hidden state solution recreated the empirically observed probe rating transition probabilities better than two hidden states. Correlations between observed transition probabilities and simulated transition probabilities for 5000 sets of 350 simulated sequences of 36 probe ratings were larger on average for the three hidden state solution (mean $r=.907, S D=.008$, range $=.872-.933$ ), and the four state solution (mean $r=.970, S D$ $=.004$, range $=.956-.983)$, than the two state solution (mean $r=.639, S D=.020$, range $=.554-.701$ ).

Supplementary Table 2 provides initial probabilities, transition probabilities, and emission probabilities for the three hidden state Markov model.

Transition probabilities for hidden states are depicted in Supplementary Figure 2 b. All hidden states were highly likely $(\sim 95 \%)$ to transition to themselves. Hidden State 1 was characterized by a large probability of emitting probe ratings of $1(72.40 \%)$ and $2(20.47 \%)$. Hidden State 1 thus reflects the second most frequently occurring $(33.41 \%$ of all probes belonged to this state based on the most probable hidden paths) hidden state in which individuals infrequently report off-task probe ratings. When Hidden State 1 occurred, it had an average duration of 14.78 probes $(S D=12.32$, range $=1-$ 36). Hidden State 2 was characterized by the likelihood of emitting a range of probe ratings of $2(40.48 \%), 3(35.90 \%)$, and $4(13.18 \%)$. Hidden State 2 was the most common state (48.95\% of all probes) in which individuals are likely to report mild to moderate attentional fluctuations. Hidden State 2 had an average duration of 16.15 probes $(S D=11.62$, range $=1-36$ ). Hidden State 3 was characterized by high likelihood of emitting probe ratings of $5(27.33 \%)$, and 6 $(60.46 \%)$. Finally, Hidden State 3 was the least frequent state (17.64\% of all probes) and had an average duration of 13.42 probes $(S D=9.90$, range $=1-36)$. Individuals on average experienced $1.98(S D=0.71,95 \%$ CI $[1.909,2.057])$ unique hidden states and had $1.38(S D=1.24,95 \%$ CI [1.252, 1.509]) transitions between distinct occurrences of hidden states.

Supplementary Figure 2d depicts the most probable hidden paths for all participants compared to observed probe ratings. Visual inspection of the most probable hidden paths suggested a clear time-ordered decrease in the overall prevalence of Hidden State 1 and corresponding increase in the frequency of Hidden States 2 and 3. We statistically 
Supplementary Table 2: Three state hidden Markov models for Goller et al. (2020) and Wiradhany et al. (2019)

\begin{tabular}{|c|c|c|c|c|c|c|c|c|c|c|c|}
\hline \multirow[b]{2}{*}{ Model } & \multirow[b]{2}{*}{$\begin{array}{l}\text { Initial } \\
\text { Prob. }\end{array}$} & \multicolumn{3}{|c|}{ Transition Prob. } & \multicolumn{7}{|c|}{ Emission Prob. } \\
\hline & & 1 & 2 & 3 & 1 & 2 & 3 & 4 & 5 & 6 & 7 \\
\hline \multicolumn{12}{|c|}{ Goller et al. (2020) } \\
\hline State 1 & 0.647 & 0.934 & 0.063 & 0.003 & 0.724 & 0.205 & 0.056 & 0.009 & 0.006 & & \\
\hline State 2 & 0.353 & 0.018 & 0.950 & 0.033 & 0.084 & 0.405 & 0.359 & 0.132 & 0.020 & & \\
\hline State 3 & 0.000 & 0.006 & 0.035 & 0.958 & 0.019 & 0.018 & 0.085 & 0.273 & 0.605 & & \\
\hline \multicolumn{12}{|c|}{ Wiradhany et al. (2019) } \\
\hline State 1 & 0.545 & 0.909 & 0.084 & 0.006 & 0.768 & 0.178 & 0.045 & 0.000 & 0.001 & 0.003 & 0.005 \\
\hline State 2 & 0.369 & 0.021 & 0.933 & 0.046 & 0.091 & 0.523 & 0.287 & 0.079 & 0.020 & 0.000 & 0.000 \\
\hline State 3 & 0.086 & 0.008 & 0.030 & 0.961 & 0.031 & 0.040 & 0.265 & 0.251 & 0.232 & 0.109 & 0.072 \\
\hline
\end{tabular}

Note: Initial starting probabilities, transition probabilities among hidden Markov states, and emission probabilities of each hidden state emitting probe ratings (1 “on-task" to 5 "off-task" or 1 "on-task" to 7 “off-task") are given for three state Markov models for studies by Goller et al. (2020) and Wiradhany et al. (2019). The Bayesian Information Criterion (BIC) is 31,242.22 and 8,194.066 for each model, respectively.

examined these changes using generalized linear mixed effects models to fit a cumulative logit proportional-odds model to the categorical state data. Probe number was included as a fixed effect to estimate linear changes in the odds of state occurrences, random participant intercepts were included, and parameters were estimated using maximum likelihood estimation based on adaptive quadrature. The odds of occurrence of Hidden State 1 (intercept $\log b=0.968$, $95 \%$ CI $[0.541,1.396])$, and the cumulative odds of Hidden State 1 and 2 (intercept $\log b=6.420,95 \%$ CI [5.963, $6.877])$, were reduced over the course of the task ( $\log b=$ $0.136, p<.001,95 \%$ CI $[-0.142,-0.131])$. Because the decrease in frequency of Hidden State 1 appeared to asymptote towards the end of the task, we also tested whether a quadratic trend could explain reductions in the prevalence of Hidden State 1 over the course of the task. The odds of occurrence of Hidden State 1 (intercept $\log b=$ $1.381,95 \%$ CI $[0.942,1.820])$, and the cumulative odds of Hidden State 1 and 2 (intercept $\log b=6.866,95 \%$ CI [6.395, 7.338]), were reduced over the course of the task (log $b=-0.211, p<.001,95 \%$ CI $[-0.230,-0.192])$ but a significant quadratic trend showed that this linear change slowed over time $(\log b=0.002, p<.001,95 \%$ CI [0.002, $0.003])$. These changes indicate overall reductions in the proportion of Hidden State 1 over time and corresponding increases in Hidden States 2 and 3.

Because attentional probes were paired with questions about thought content, we aggregated the kinds of thought content according to the most probable hidden state that occurred during each experience sampling probe. Supplementary Figure 3 depicts the overall proportion of thought content occurring during each hidden state. A significant $\chi 2$ test of independence, $\chi^{2}(10)=4380.80, p<.001$, demonstrated that the distribution of thought content depended on the corresponding attentional probe hidden state. Individuals tended to report that they experienced thoughts related to the task and those related to evaluation of their task performance during Hidden State 1. Individuals reported a range of task related and evaluative thoughts during Hidden State 2, and primarily neutral task-unrelated thoughts. Hidden State 3 on the other hand primarily accompanied neutral and affectively valenced task-unrelated thoughts.

\section{Secondary Analysis of Wiradhany et al. (2019)}

Frequency distribution of attentional probe ratings. Supplementary Figure 4a depicts the sequences of attentional probe ratings (from 1 "on-task" to 7 "off-task") during the working memory change-detection task for all individuals. The distribution of all 3,166 attentional probe ratings (see Supplementary Figure 4b) was strongly positively skewed with $34.30 \%$ of all attentional probe ratings rated equal to 1 (on-task). $65.70 \%$ of all probes were rated greater than 1 . $8.65 \%$ of probes were rated 5 or greater, and $1.64 \%$ of all probes were rated 7 (off-task). Participants on average used $3.42(S D=1.26,95 \%$ CI $[3.570,3.265]$, range $=1-7)$ unique categories out of 7 categories. Participants, as a whole, used the range of responses to categorize their current attentional state. Individuals were thus highly likely to report being ontask to some extent (i.e., a probe rating of 1 or 2) compared to reporting being off-task.

\section{Transition Probabilities of attentional probe ratings.}

Markov-chain transition probabilities for attentional probe ratings revealed strong transition probabilities along the diagonal of the transition probability matrix. Supplementary Figure $4 \mathrm{c}$ depicts the transition probability matrix of all attentional probe ratings. 
a.

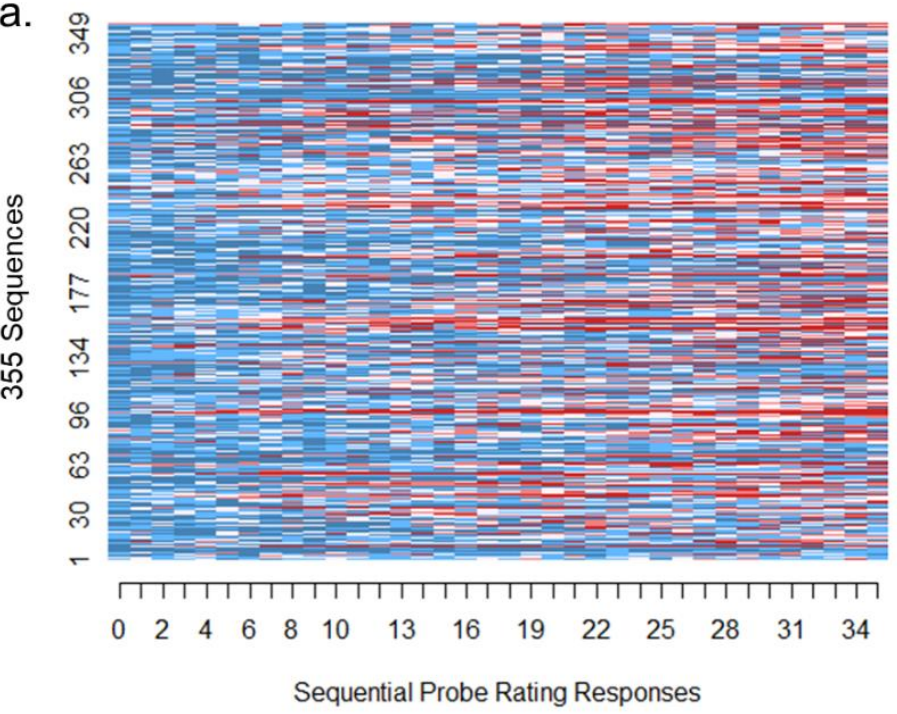

b.

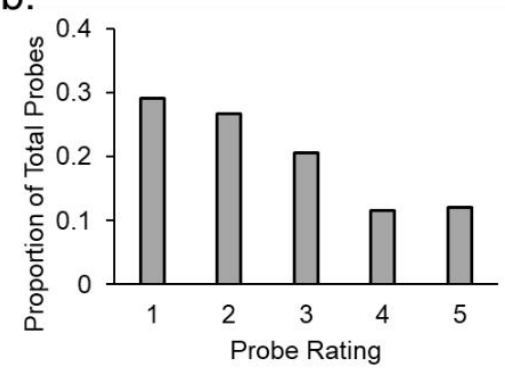

C.

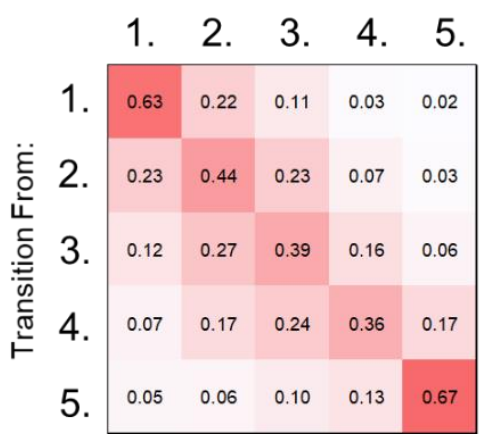

Supplementary Figure 1: Sequences of 36 probe ratings (rated from 1 "on-task" to 5 "off-task") during the SART are depicted (a.) on rows for each individual $(n=355)$ for the study by Goller et al. (2020). The distribution of all 12,780 probe ratings (b.) and the transition probability matrix of probe ratings (c.) are shown for sequences of probe ratings.

a.

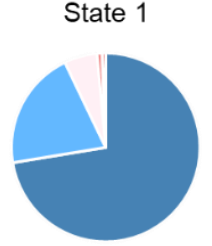

$64.70 \%$
State 2

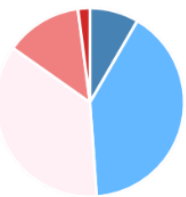

$35.30 \%$
State 3

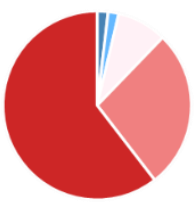

$0 \%$

\begin{tabular}{ll|} 
1. On-Task & $\square$ 3. Both On-Task \& Off-task $\square$ 5. Off-task \\
2. Mostly On-task & $\square$ 4. Mostly Off-task
\end{tabular}

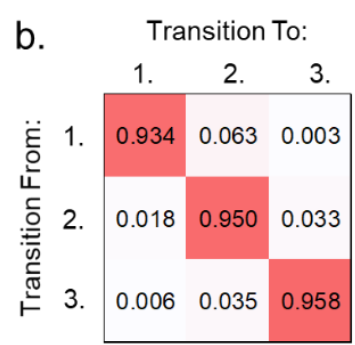

C.

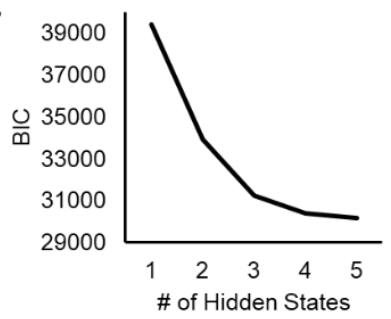

d.
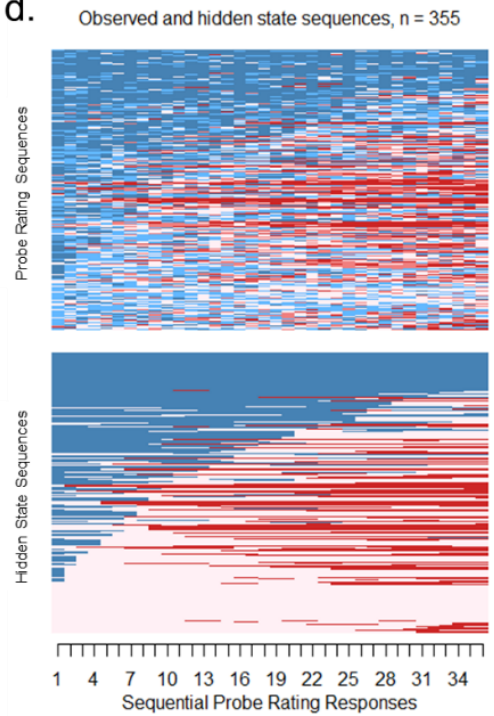

Supplementary Figure 2: Three hidden Markov states were identified as the optimal model for the study by Goller et al. (2020). Each state is depicted (a.) as a circle chart in which the emission probabilities for probe ratings are given as proportions. Initial state probabilities are indicated below each circle chart. Transition probabilities between hidden states are indicated (b.) in the transition matrix. The Bayesian Information Criterion (BIC) is given (c.) for hidden Markov models with different numbers of hidden state solutions. Sequences are sorted (d.) according to multidimensional scaling of hidden state sequence dissimilarities in order to group sequences on rows according to their similarity to illustrate the most probable hidden Markov paths underlying the probe rating responses. 


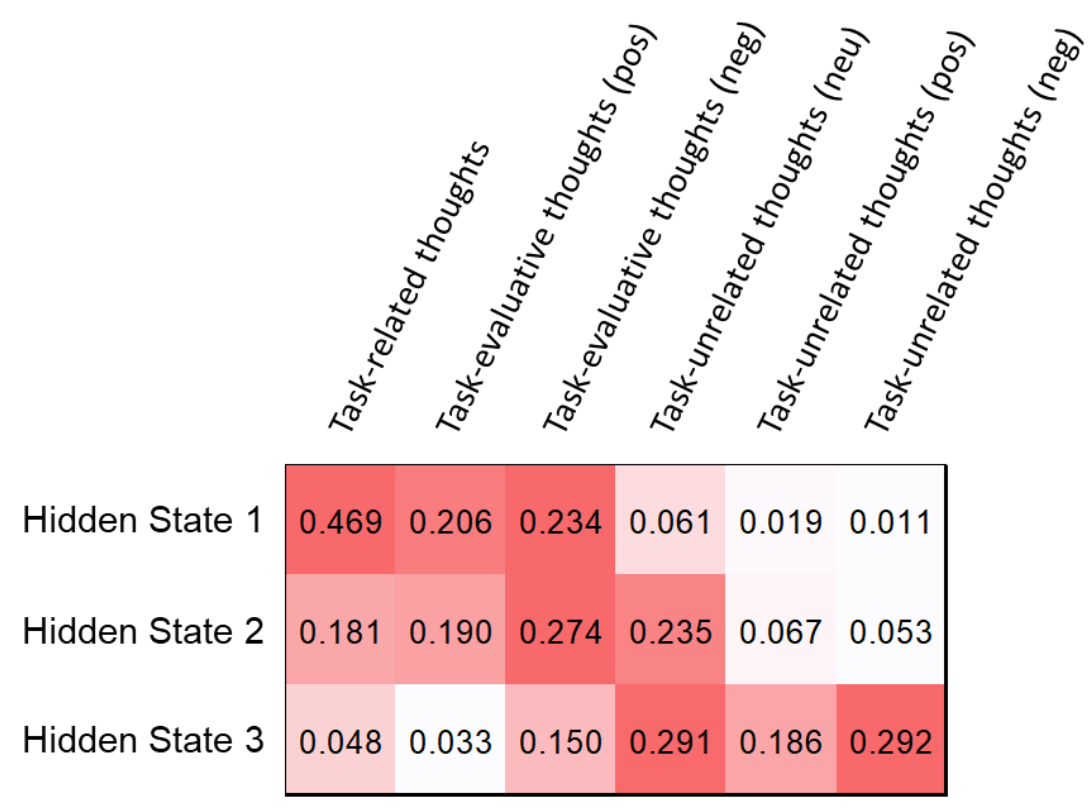

Supplementary Figure 3: The proportion of thought content probes are depicted according to the most probable hidden state of their paired attentional probe rating.

Participants on average had $5.81(S D=2.72,95 \%$ CI $[5.483$, 6.142]) transitions between distinct probe ratings. Transitions were more likely to occur than chance (i.e., transition probabilities more extreme than $97.5 \%$ of 5,000 random permutations of probe rating sequences) for transitions between ratings of $1 \rightarrow 1,2 \rightarrow 2,3 \rightarrow 3,4 \rightarrow 4,5 \rightarrow 5,6 \rightarrow$ 6 , and $7 \rightarrow 7$. Transitions were also more likely than chance to occur for transitions between ratings of $6 \rightarrow 7$. Individuals were also likely $(63.8 \%)$ to remain in a highly focused state following a probe rating of 1 , as transitions from ratings of 1 $\rightarrow 3,1 \rightarrow 4$, and $1 \rightarrow 6$, occurred less frequently than expected by chance alone. Individuals were less likely than chance to transition from ratings of $2 \rightarrow 1$, and $3 \rightarrow 1$.

Following a complete attentional lapse, individuals were highly likely $(41.0 \%)$ to remain in that same off-task state (i.e., probe rating of 7) during the next probe question, and were less likely than chance to transition to more on-task states for transitions between ratings of $6 \rightarrow 2$. Transitions were also less likely than chance to occur for transitions between ratings of $2 \rightarrow 7,3 \rightarrow 6,4 \rightarrow 6$, and $5 \rightarrow 6$. All other transitions were no more or less extreme compared to chance alone.

Hidden Markov states. Three hidden states were identified as optimal. Each of the three hidden states are shown in Figure $5 \mathrm{a}$ as a circle chart in which the emission probabilities are depicted as proportions. Additional hidden states did not greatly reduce the BIC of the model (see Supplementary Figure 5c). The three hidden state solution also recreated the empirically observed transition probabilities better than two hidden states. Correlations between observed transition probabilities and transition probabilities for 5,000 sets of 300 simulated sequences of 12 probe ratings were larger on average for the three hidden state solution (mean $\mathrm{r}=.794, S D$ $=.027$, range $=.699-.879)$, and the four state solution (mean $r=.927, S D=.023$, range $=.802-.979)$, than the two state solution (mean $r=.603, S D=.013$, range $=.554-$ .653). Supplementary Table 3 provides initial probabilities, transition probabilities, and emission probabilities for the three hidden state Markov model.

Transition probabilities for hidden states are depicted in Supplementary Figure 5b. All hidden states were highly likely $(\sim 93 \%)$ to transition to themselves. Hidden State 1 was characterized by a large probability of emitting probe ratings of $1(76.78 \%)$ and $2(17.80 \%)$. Hidden State 1 thus reflects the second most frequently occurring $(38.89 \%$ of all probes belonged to this state based on the most probable hidden paths) hidden state in which individuals infrequently report off-task probe ratings. When Hidden State 1 occurred, it had an average duration of 7.34 probes $(S D=4.29$, range $=1-$ 12). Hidden State 2 was characterized by the likelihood of emitting a range of probe ratings of $2(52.30 \%), 3(28.66 \%)$, and $4(7.88 \%)$. Hidden State 2 was the most common state (44.99\% of all probes) in which individuals are likely to report mild to moderate attentional fluctuations. Hidden State 2 had an average duration of 8.14 probes $(S D=3.77$, range $=$ 1-12). Hidden State 3 was characterized by high likelihood of emitting a range of probe ratings of $3(26.48 \%), 4$ $(25.11 \%), 5(23.18 \%), 6(10.90 \%)$, and 7 (7.19\%). Finally, State 3 was the least frequent state $(16.12 \%$ of all probes) and had an average duration of 8.15 probes $(S D=3.58$, 


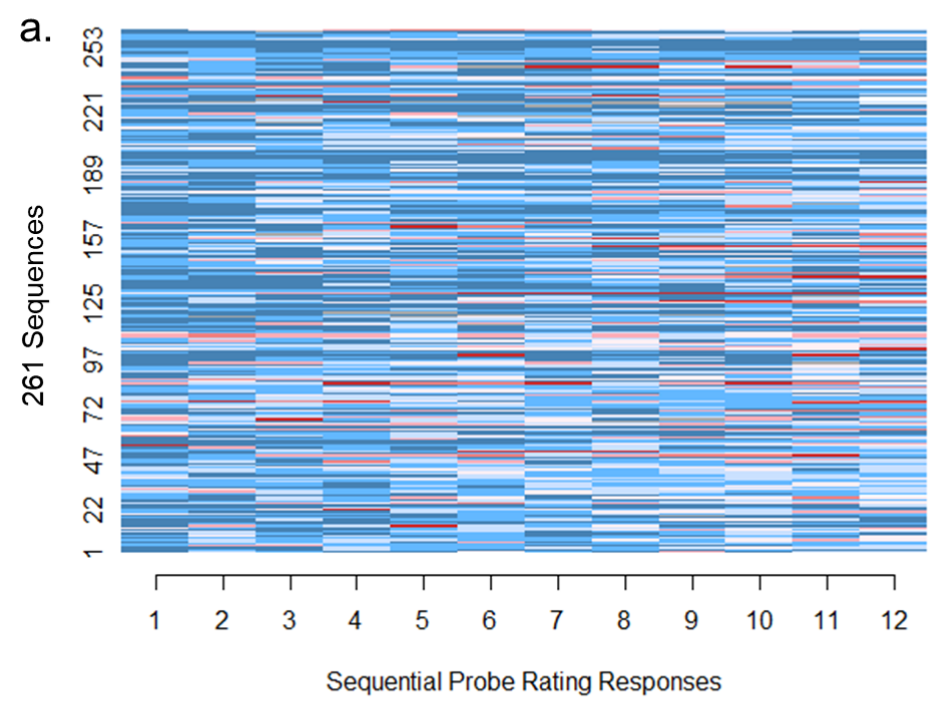

\begin{tabular}{|lll|}
\hline$\square$ 1. On-task & $\square$ 4. Both On-task \& Off-task \\
$\square$ 2. Moderately On-task & $\square$ 5. Off-task \\
$\square$ 3. Slightly On-task & $\square$ 6. Moderately Off-task & \\
\hline
\end{tabular}

b.

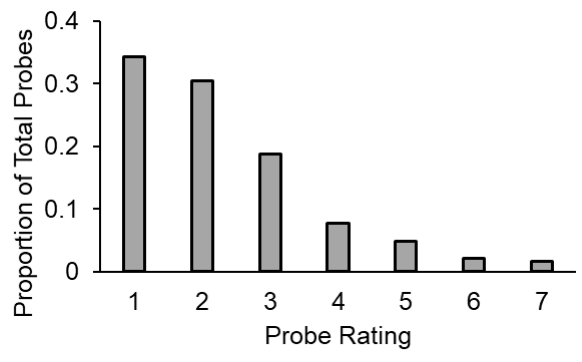

C.

Transition To:

\begin{tabular}{|c|c|c|c|c|c|c|c|}
\hline & 1. & 2. & 3. & 4. & & 6. & 7. \\
\hline 1. & 0.64 & 0.23 & 0.09 & 0.01 & 0.02 & 0.00 & 0.00 \\
\hline 2 & 0.23 & 0.45 & 0.22 & 0.06 & 0.03 & 0.01 & 0.00 \\
\hline 3. & 0.12 & 0.33 & 0.32 & 0.14 & 0.07 & 0.01 & 0.01 \\
\hline 4. & 0.09 & 0.19 & 0.30 & 0.26 & 0.12 & 0.02 & 0.02 \\
\hline 5. & 0.07 & 0.12 & 0.25 & 0.20 & 0.22 & 0.12 & 0.01 \\
\hline 6 & 0.06 & 0.02 & 0.17 & 0.13 & 0.15 & 0.33 & 0.15 \\
\hline & 0.13 & 88 & 0.10 & 0.08 & 0.05 & 0.15 & 41 \\
\hline
\end{tabular}

Supplementary Figure 4: Sequences of 12 probe ratings (rated from 1 "on-task" to 7 "off-task") following blocks of trials are depicted (a.) on rows for each individual $(\mathrm{n}=261)$ for the study by Wiradhany et al. (2019). The distribution of all 3,166 probe ratings (b.) and the transition probability matrix of probe ratings (c.) are shown for sequences of probe ratings. a.

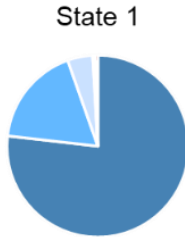

$54.52 \%$

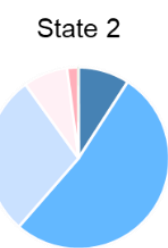

$36.91 \%$

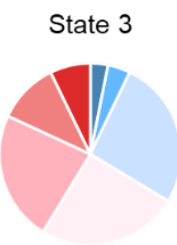

$8.57 \%$

\begin{tabular}{|lll|}
\hline$\square$ 1. On-task & $\square$ 4. Both On-task \& Off-task $\square$ 7. Off-task \\
$\square$ 2. Moderately On-task & $\square$ 5. Slightly Off-task & $\square$ missing \\
$\square$ 3. Slightly On-task & $\square$ 6. Moderately Off-task & \\
\hline
\end{tabular}

b. Transition To:

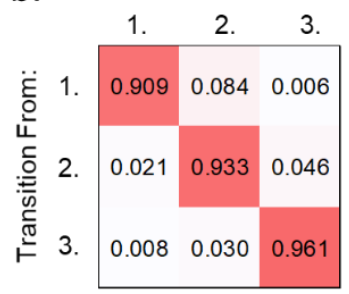

c.

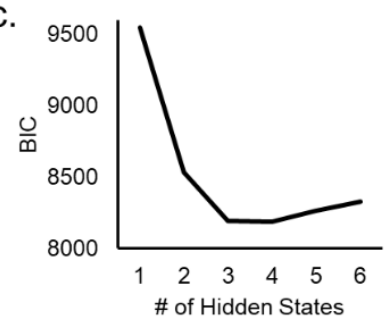

d.
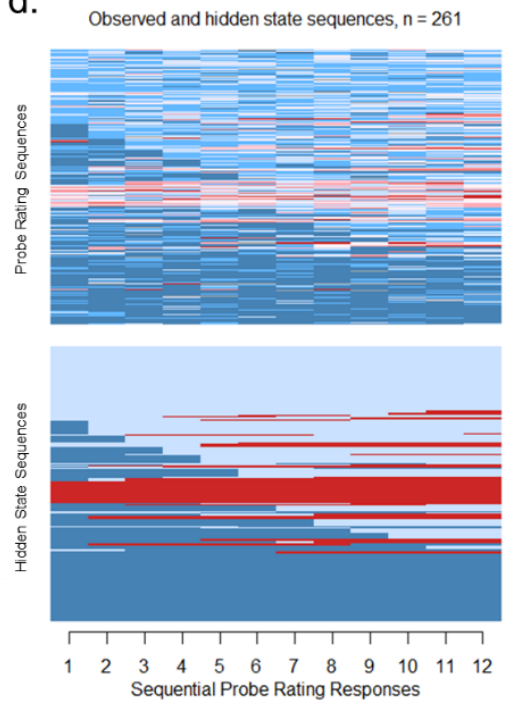

Supplementary Figure 5: Three hidden Markov states were identified as the optimal model for the study by Wiradhany et al. (2019). Each state is depicted (a.) as a circle chart in which the emission probabilities for probe ratings are given as proportions. Initial state probabilities are indicated below each circle chart. Transition probabilities between hidden states are indicated (b.) in the transition matrix. The Bayesian Information Criterion (BIC) is given (c.) for hidden Markov models with different numbers of hidden state solutions. Sequences are sorted (d.) according to multidimensional scaling of hidden state sequence dissimilarities in order to group sequences on rows according to their similarity to illustrate the most probable hidden Markov paths underlying the probe rating responses. 
range $=1-12)$. Individuals on average experienced 1.50 (SD $=0.61,95 \%$ CI $[1.425,1.572])$ unique hidden states and had $0.54(S D=0.66,95 \%$ CI $[0.456,0.617])$ transitions between distinct occurrences of hidden states.

Supplementary Figure 5d depicts the most probable hidden paths for all participants compared to observed probe ratings. Visual inspection of the most probable hidden paths also suggested a time-ordered decrease in the overall prevalence of Hidden State 1 and increase in other states. We fit a cumulative logit proportional-odds model to the categorical state data. The odds of occurrence of Hidden State 1 (intercept $\log b=0.897,95 \%$ CI $[0.147,1.646])$, and the cumulative odds of Hidden State 1 and 2 (intercept $\log b=$ $7.275,95 \%$ CI $[6.410,8.141])$, were reduced over the course of the task $(\log b=-0.368, p<.001,95 \%$ CI [-0.409, -

$0.327])$. These changes indicate overall reductions in the proportion of Hidden State 1 over probes and corresponding increases in Hidden States 2 and 3. 